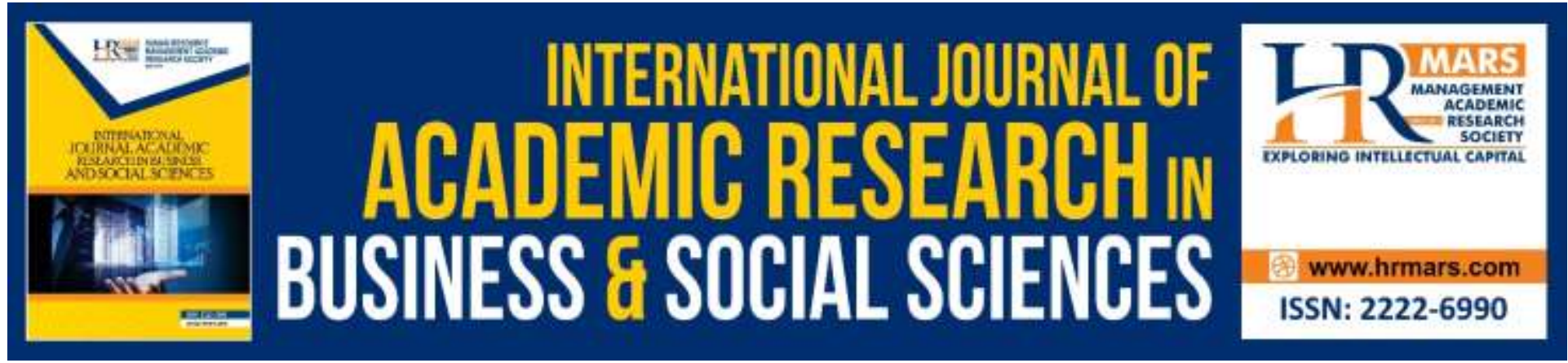

\title{
Profiling of Manufacturing Industries Bumiputera Entrepreneurs (MIBE) Government Related Agencies
}

\author{
M. S. Hamid, S. B. Mohamed, A. A. Abdullah
}

To Link this Article: http://dx.doi.org/10.6007/IJARBSS/v9-i7/6240

DOI: $10.6007 /$ IJARBSS/v9-i7/6240

Received: 22 May 2019, Revised: 27 June 2019, Accepted: 30 June 2019

Published Online: 23 July 2019

In-Text Citation: (Hamid, Mohamed, \& Abdullah, 2019)

To Cite this Article: Hamid, M. S., Mohamed, S. B., \& Abdullah, A. A. (2019). Profiling of Manufacturing Industries Bumiputera Entrepreneurs (MIBE) Government Related Agencies. International Journal of Academic Research in Business and Social Sciences, 9(7), 1212-1262.

Copyright: (C) 2019 The Author(s)

Published by Human Resource Management Academic Research Society (www.hrmars.com)

This article is published under the Creative Commons Attribution (CC BY 4.0) license. Anyone may reproduce, distribute, translate and create derivative works of this article (for both commercial and non-commercial purposes), subject to full attribution to the original publication and authors. The full terms of this license may be seen at: http://creativecommons.org/licences/by/4.0/legalcode

Vol. 9, No. 7, 2019, Pg. 1212 - 1262

http://hrmars.com/index.php/pages/detail/IJARBSS

JOURNAL HOMEPAGE

Full Terms \& Conditions of access and use can be found at http://hrmars.com/index.php/pages/detail/publication-ethics 


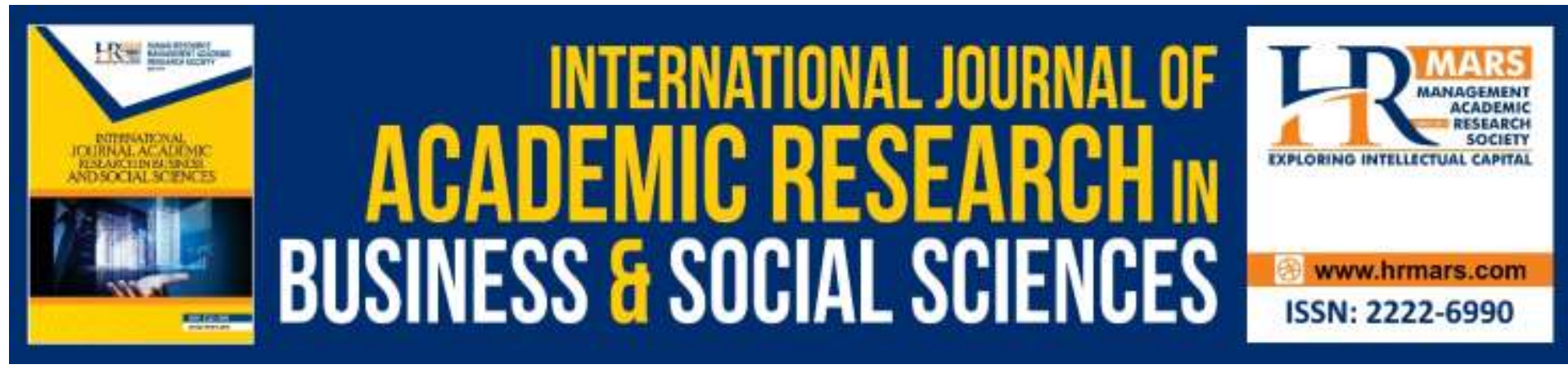

\title{
Profiling of Manufacturing Industries Bumiputera Entrepreneurs (MIBE) Government Related Agencies
}

\author{
M. S. Hamid ${ }^{\mathrm{a}}$, S. B. Mohamed ${ }^{\mathrm{a}}$, A. A. Abdullah ${ }^{\mathrm{b} *}$ \\ a Faculty of Innovative, Design, and Technology (FRIT), Universiti Sultan Zainal Abidin, Gong Badak \\ Campus, 21300 Kuala Terengganu, Malaysia, ${ }^{b}$ Fakulti of Economy and Management Sciences \\ Department Universiti Sultan Zainal Abidin, Gong Badak Campus, 21300, Kuala Terengganu, \\ Malaysia. \\ Email: azizabdullah@unisza.edu.my, sobriarie@yahoo.com, saifulbahri@unisza.edu.my
}

\begin{abstract}
A SME advancement programs was implemented by the governance of Malaysia and supervised through the 24 ministries. It was purposely aim to determine which agencies that give contribution to the businessperson. Similarly, SME Integrated Plan of Action (SMEIPA) refer to the document containing the yearly report on the status, analysis, assessment and how successful the plans enacted by all parties and ministries. The execution of diverse plans has been assigned to some agencies and department of the government as well. Hence, the aim of this research is to recognize the agency of Malaysia's Government in pursuance of elucidating the degree of success upon support granted by the government towards the enterprisers of Bumiputera. An evaluation via online survey was carried out among the 24 Ministries. A notable revelation is that these constituted agencies was purposed to recommend supplementary service for instance the Canter of Export Trade with the role in discloses the enterprisers to international chances, the Canter of National Productivity assigned with supplying guidance in accordance with management, which are executed in association with Research Development Association of National Entrepreneurship together with the Canter of Malaysian Entrepreneur Development. Last but not least, Bumiputera Entrepreneur accomplishment would be qualified to alter the bias intention of entrepreneurial ethnicity from another race. In realities, the identification of those party purposes collateral with government intentions to enhance the entrepreneurs' number involving Bumiputera is a great initiative with regards to increase the GDP in Malaysia.
\end{abstract}

Keywords: Sustainable Entrepreneurs, Bumiputera, Profiling, Malaysia Agency.

\section{Introduction}

The Malays in these states are the dominant and most relevant in the history of the Malaysian entrepreneurship development. They are commonly known to be Muslims engrossed in their customs (adats) and cultures, enjoying the inalienable rights and privileges of the Bumiputera ordained for them under the constitution (Constitution, 2010). These rights were conferred on the Malays being the indigenous people in Malaysia so as to compensate them for the imposition of the other immigrant races of Chinese and Indians on them by the British colonists. These other races had a massive entry into Malaysia during the early 20th century as a result of the boom in the tin and rubber production in Malaysia, and subsequently, got citizenship status during the independence era (Alom, Abdullah, 
Moten, \& Azam, 2016; Shome \& Hamidon, 2009). Due consideration is given to entrepreneurship by policy makers in several countries or international corporations in promoting and enhancing the entrepreneurial climate. They formulate viable policies to boost the entrepreneurial development either by uprooting bottlenecks or some forms of palliatives designed to tackle some menace (Ahmad \& Hoffman, 2007). This had been a trend witnessed since around the mid-20th century in developed nations as US, UK, Australia, as well as in developing nations.

\section{Literature Review}

Hence, no doubt that in order to address the growing discontentment among the races, and revamp the marginalized Bumiputera society under the colonial period, the Malaysian government initiated some developmental policies. Hence, since 1970s, through the various policies: New Economic Policy (1970 - 1990); National Development Policy (NDP) (1991 - 2000); National Vision Policy - NVP (2001 2005); National Mission Policy (NMP) (2006 - 2020), which later incorporated the Malaysian National Development Strategy (MyNDS) (2015 - 2020); billions of Malaysian ringgits were spent on programs and initiatives created to cater for the Bumiputera entrepreneurship development.

Most SMEs requires support or assistance from the government to be more competitive in the global business environment. The role of government is to encourage companies to move to higher levels and gain competitive advantage in the globalized environment (Lukas \& Basuki, 2015). SMEs has become the backbone of the world economy as they take a huge function in employment, whereas, the strength, excellence, achievement and success of the SMEs are said to be key indicators in evaluating an economy's growth and potential development (Wiklund, 1998). The basic entrepreneurial activity contributed by SMEs has been widely acknowledged as the motivating factor for innovation and economic growth. Therefore, the achievement or otherwise of SMEs as well as entrepreneurship remains paramount to government and policy makers (Schaper, 2010).

Follow to Hashim, Che, Hamzah, \& Umar, (2018), the presence of Malay attitudes and belief systems which appeared to be least desirable to a business culture, and which had been reinforced over the years by feudalism and, to a certain extent, colonialism. Entrepreneurship development assumed much greater significance in Malaysia after the 1969 riot between the main races Bumiputera or Malays, Chinese and Indians as the non-Bumiputera (Sabiu, Abdullah, \& Amin, 2017). There were serious concerns about the wide income disparity between the Bumiputera and the non-Bumiputera. The former were mostly restricted to agriculture under the pre-independence colonial rule and lacked entrepreneurial inclination. While, the latter had economic ascendancy and a long history of entrepreneurship which provided them a significant income (Ariff et al., 2015; Awang et al., 2009). However, although Malay elites dominated and controlled the administrative system, they did not have corresponding economic power (Chan, Paul, Horii, \& Kenzo, 1986).

For their part, the Malay petite bourgeoisie were faced with inadequate capital, skilled labour shortages, which in turn impeded their success (Whah \& Guan, 2017). Due to these constraints, ambitious Malay businessmen, politicians and bureaucrats turned to economic and political ethnonationalism in the first Bumiputera Economic Congress (BEC) in 1965. This was a significant milestone that marked a turning point in the development of Malay capitalism. The demand for a more active role by the state in support of Malay commercial expansion continued and was the reason behind the organization of the second BEC in 1968.

In response to the first BEC, the government established the first Malay commercial bank (Bank Bumiputera) in 1965 to: provide Malays with increased capital; distribute shares to Malays and Malay 
interests and break Chinese dominance of the banking industry (Whah \& Guan, 2017; Thoburn \& Snodgrass, 2006). In 1966, the central bank, Bank Negara, seized the opportunity and took over Malayan Banking Berhad, a Chinese-controlled bank facing difficulties (Ranjit, 1987). In the same year, the Rural and Industrial Development Authority (RIDA) which had been established in 1950 by the colonial government to cultivate a Malay petite bourgeoisie and look after rural development was replaced with Majlis Amanah Rakyat (MARA) or Council of Trust for Indigenous People and given greater resources. Recapitulate, prior to the BEC, the first two strategies used to develop a Malay industrial and commercial bourgeoisie were: protection through the imposition of Malay quotas on business licenses, employment and education; and assistance in the form of credit, training and business premises channelled through state-owned enterprises. There was no direct intervention, as the state only encouraged Malays to accumulate capital through these two strategies.

Nevertheless, the first and second BECs in 1965 and 1968 marked the transition from a facilitative role to direct state intervention for the Malays and Malay interests (Chan et al., 1986). The state's direct intervention from late 1969 onwards through public enterprises characterizes a major change from laissez faire to a develop mentalist role, namely to address the "Malay dilemma" through creating a new business and managerial class by modernizing Malay society Abdul Rahman 1996

Hence, Amir, (1983) the task of assisting the development of indigenous Bumiputera entrepreneurs was at the beginning entrusted to the Malaysian Civil Service (MCS) and later extended to include other quasi-government agencies or government-backed agencies. Unequivocally, the Malaysian government made it paramount to redress the issue and bridge the growing discontent about the economic inequalities between the races. Therefore, the New Economic Policy (1970 - 1990) was implemented serving the main objectives of: restructuring the society in general by enhancing the economic activities of all races and poverty eradication especially for the Bumiputera. And importantly, the other is developing the entrepreneurial capabilities of the Bumiputera in the commercial arena (Koon, 1997). It thus created the Bumiputera Commercial and Industrialization Community $(\mathrm{BCIC})$. The aim was on nurturing as well as boosting active Bumiputera participation in lucrative entrepreneurial activities at all levels where the business domain was predominated by the non-Bumis. And, another goal was for the Bumiputera to equally achieve equity ownership of at least $30 \%$ by 1990 (Ariff \& Abubakar, 2007).

To do this, the government concentrated focus on the creation of trust agencies and their subsidiaries. These agencies include National Entrepreneurship Institute, SME Bank, Bank Rakyat, National Corporation Council or Perbadanan Nasional Berhad (PNS) People's Trust Council or Majlis Rakyat Amanah (MARA), National Equity Corporation or Permodalan Nasional Berhad (PNB), Urban Development Authority (UDA), Tabung Economi Kumpulan Usahawan Niaga (Tekun) for loans to Bumiputra entrepreneurs, Contractor Service Centre (PKK) to help Bumiputra contractors, State Economic Development Corporations (SEDCs), among others. These establishments were made to promote share ownership, and open business opportunities for Bumiputera to fully involve in creation and management of the country's wealth (Berita, 2008; Buang \& Yusof, 2006).

\section{New Economic Policy (NEP) (1971 - 1990)}

According to Economic Planning Unit, (2001), by the 1990s after 20 years of the NEP implementation, the first objective made tremendous achievement. Basically, poverty was reduced by more than $200 \%$ from 1970 to 1990. Meanwhile, the second goal of developing economic imbalances and boosting Malay entrepreneurship could not achieve much. Thus, the mixed success outcome of the NEP 
propelled the government to integrate the Bumiputera entrepreneurship development into Malaysia's subsequent development policies (Shome \& Hamidon, 2009; Sabiu et al., 2017).

\section{National Development Plan (NDP) (1991 - 2000)}

Subsequently, the National Development Policy (NDP) (1991 - 2000) focused on the Bumiputera participation in the private sector. Various privatization programs, business and wealth creation management, training and equally the achievement of at least $30 \%$ contract works in privatized project for the Bumiputera contractors were implemented. To augment the various programs, the Ministry of Entrepreneurship Development was established in 1995 to support the development of Bumiputera entrepreneurs as well as coordinate entrepreneurship activities in general (Ariff \& Abubakar, 2015). A significant outcome of the policy witnessed, was the increase in the number of Bumiputera enterprises with an estimate of 697,900 sole proprietorship and partnership companies. While about 57,700 private limited companies registered with Registrar of Business (ROB) and Registrar of Company (ROC) respectively. Even though, this achievement was small compared to that of their non-Bumi counterparts, and equally lacked technological as well as management competencies (Anthony \& Syahira, 2009).

\section{National Vision Policy (NVP) (2001 - 2004)}

In the same vein, the National Vision Policy - NVP (2001 - 2005) incorporated the achievement of Bumiputera equity ownership of at least $30 \%$ by the end of the decade. And the policy also focused on effective involvement of the Bumiputera in the prospering sectors in the economy (OPP3, 2001). However, with the change of new political administration in 2004, the NVP metamorphosed into the National Mission Policy (NMP) $(2006$ - 2020). This policy stressed on achieving effective Bumiputera as well as $30 \%$ ownership by 2020 and enhancing greater income and wealth distribution (Hamidon, Suhaimie, Yunoh, Naqib, \& Hashim, 2017; OPP3, 2001).

For instance, about 82 microcredit schemes were offered through some of the above agencies for SMEs to access non-collateral loans varying from RM500 to RM50,000 (Rostamzadeh, Ismail, \& Bodaghi Khajeh Noubar, 2014). As mentioned in Muhammad Khalique, Abu Hassan Md Isa, Jamal Abdul Nassir Shaari, \& Adel Ageel, (2011) from RM 1,561.6 million in the Eight Malaysian Plan, it drastically increased to RM 2,160.2 million for the development of SMEs in the Ninth Malaysian Plan (9MP) (2006-2010). Notwithstanding this huge allocation, the government established other support programs, institutions and agencies to complement its effort. These efforts include providing guidance, promotion, production efficiency, R\&D program and product development (Mohamed, Rezai, Shamsudin, \& Mahmud, 2012). According to MECD (2007) various training programs, workshops, seminars, expositions were delivered to promote entrepreneurial interest among the Bumiputera, and equip them well for local and international competition (Muhammad et al., 2011).

\section{National Mission Policy (NMP) (2005 - 2015)}

Equally, it is noteworthy that under the 9MP, the Ministry of Entrepreneurship and Cooperative Development (MECD) allocated RM 5.77 billion budget ceiling to execute some developmental projects of about 633 in number (Hamidon, 2009). These projects majorly bordered on inculcating entrepreneurship culture in students, graduates, youth and women of Bumiputera totalling 1.5 million. The development of 150,000 new Bumiputera entrepreneurs to beef up the already existing 25,000 Bumiputera enterprises and 600 cooperatives were included. Similarly, 800 new cooperatives, 50 franchisor and 1000 franchisee enterprises were to be raised. Lastly, achievement of at least $25 \%$ Bumiputras equity ownership by 2010 was prioritized too ( Hamidon, 2008). 
Ahmad Badawi became the Prime Minister in late 2003. Abdullah probably saw the increasing intraethnic wealth gap within the Malays following the past three decades of wealth redistribution that focused on large enterprise development. Developing Bumiputera entrepreneurial SMEs was a rational way to restructure society and eradicate poverty. Abdullah established the National SME Development Council (NSDC) in 2004 to support SME growth. The BCIC agenda under Abdullah was to expedite the development of self-reliant, sustainable and competitive Bumiputera entrepreneurs and the creation of strong Bumiputera SMEs enhanced through the creation of linkages and clusters among Bumiputera enterprises, between Bumiputera and non-Bumiputera, and between GLCs and Bumiputera enterprises (Whah \& Guan, 2015).

The emergence of Prime Minister Najib Razak in 2009 came with turmoil initially to the existing preferential treatment policies of the Bumiputera as he proposed to introduce a need-based strategy approach to tackling the underdevelopment of the bottom 40 rung of the society irrespective of his/her race. Though, this course was later struck due to overwhelming protest by politicians, and was replaced to another project that led to the establishment of Teraju, which focuses on the boosting of economic participation of Bumiputera in lucrative sectors of the economy (Chin \& Dosch, 2015).

Eventually, the Tenth Malaysian Plan (10MP) (2011 - 2015), encapsulated the spirit of 1Malaysia to ensure equitable access to economic participation for all Malaysians and enable equitable opportunities for all in the area of capacity building and encourage innovation-driven entrepreneurship. It is aimed at enhancing Bumiputera economic participation and strengthening their entrepreneurship to encourage competiveness in a high impact sectors. And also, it aimed at extending wealth ownership through institutional ownership over corporate equity which enables pooling and mobilizing fund and distribution of profits to a larger group of participants (Economy, 2015).

More so, the 10MP noted that the Bumiputera agenda is concerned with growth and distribution to foster competitiveness and resilience with increased participation of the Bumiputera in highly challenging business environments. Government investment institutions like Perbadanan Nasional Berhad (PNB) and Ekuiti Nasional Berhad (EKUINAS) would support promising Bumiputera companies to prosper in their respective fields of operations (Bernama, 2018). Similarly, government's funding, support services, capacity development programs and implementation agencies are based on need based at different business stage (Economy, 2015). At micro enterprises level, funding, capacity building and infrastructure were provided to improve the livelihood of Bumiputera in the bottom $40 \%$ income earners. Also, RM 4.5 billion was allocated and more to be raised from the private sector to undertake investments in growth stage companies, including existing strong Bumiputera companies to facilitate their growth. Also, the government placed greater emphasis on scaling up and promoting innovation-intensive businesses to grow the Bumiputera Commercial Industrial Community (BCIC). As it equally provided government assistance to open new markets for the Bumiputera entrepreneurs (Economy, 2015).

\section{Malaysia National Development Strategy (MyNDS) (2015 - 2020)}

And currently, the Malaysian National Development Strategy (MyNDS) in the $11^{\text {th }}$ Malaysian Plan (2015 - 2020) RM350 million is allocated for financing 33,000 new entrepreneurs. Current status of SME accounting for $33 \%$ of GDP is targeted to be increased to $41 \%$ by 2020 , whereby the SME Investment Partner would be supported by the government. EKUINAS would be allocated with RM600 million to promote Bumiputera ownership in private companies and Government Linked Corporations (GLCs) among other things (Bernama, 2014). By December, 2015, it was reported that 512 firms of 
INTERNATIONAL JOURNAL OF ACADEMIC RESEARCH IN BUSINESS AND SOCIAL SCIENCES Vol. 9, No. 7, July, 2019, E-ISSN: 2222-6990 @ 2019 HRMARS

Bumiputera had benefitted financially up to RM61.29 million through the Bumiputera Enterprise Enhancement Programme (SME, 2016). 


\section{Research Methodology}

This study used data list of all agencies in Malaysia Government and related business agencies from SME's Corp department. The ministries that involve is, Prime Minister's; Ministry of Agriculture and Agro-based Industry; Ministry of Communication and Multimedia; Ministry of Defence; Ministry of Domestic Trade and Consumer Affairs; Ministry of Economic Affairs; Ministry of Education; Ministry of Energy, Technology, Science, Climate Change and Environment; Ministry of Entrepreneurship Development; Ministry of Federal Territories; Ministry of Finance; Ministry of Foreign Affairs; Ministry of Health; Ministry of Home Affairs; Ministry of Housing and Local Government; Ministry of Human Resources; Ministry of International Trade and Industry; Ministry of Primary Industries; Ministry of Rural Development; Ministry of Tourism, Art and Culture; Ministry of Transport; Ministry of Water, Land and Natural Resources; Ministry of Women, Family and Community Development; Ministry of Works; Ministry of Youth and Sports; and Non-ministerial bodies.

\section{Result and Discussion}

\section{Government Agencies}

The government carries out a range of SME developmental plans conducted through the 24 ministries and total of 336 agencies in Malaysia Government and 16 agencies were supported entrepreneurship (SME, 2016). Equally, SME, (2019) through the SME Integrated Plan of Action (SMEIPA) is the document presenting the annual report on the assessment, analysis, status, and how effective the programs carried out by all the ministries and the agencies. Below is the profiling of all agencies shelter under Malaysia government. This is a list of agencies of Malaysian federal government. The list includes statutory bodies, government-linked companies and organizations.

Table 1: List of All Agencies Under Malaysia Government. Retrieve from https://en.wikipedia.org/wiki/List_of_federal_agencies_in_Malaysia

\begin{tabular}{|c|c|c|}
\hline No & AGENCIES & MINISTRY \\
\hline 1. & National Palace & Prime Minister's \\
\hline 2. & $\begin{array}{l}\text { Prime Minister's Office (PMO) } \\
\text { Deputy Prime Minister's Office } \\
\text { (DPMO) } \\
\text { Chief Executive's Management } \\
\text { Information System (SMPKE) } \\
\text { Malaysian Institute of Wasatiyyah } \\
\text { (IWM) } \\
\text { National Branding Unit }\end{array}$ & Prime Minister's \\
\hline
\end{tabular}


INTERNATIONAL JOURNAL OF ACADEMIC RESEARCH IN BUSINESS AND SOCIAL SCIENCES Vol. 9, No. 7, July, 2019, E-ISSN: 2222-6990 @ 2019 HRMARS

\begin{tabular}{|c|c|c|}
\hline & $\begin{array}{l}\text { Socioeconomic Development of } \\
\text { Indian Community Unit (SEDIC) } \\
\text { Special Implementation Task Force } \\
\text { (SITF) } \\
\text { Special Secretariat for the } \\
\text { Empowerment of Indian } \\
\text { Entrepreneurs (SEED) }\end{array}$ & \\
\hline 3. & Chief Secretary's Office (Website) & Prime Minister's \\
\hline 4. & General Administration & Prime Minister's \\
\hline 5. & Advisory Board & Prime Minister's \\
\hline 6. & Attorney General's Chamber (AGC) & Prime Minister's \\
\hline 7. & $\begin{array}{l}\text { Cabinet, Constitution } \\
\text { Intergovernmental Affairs } \\
\text { (BKPP) }\end{array}$ & Prime Minister's \\
\hline 8. & $\begin{array}{l}\text { Department of National Unity and } \\
\text { Integration (JPNIN) }\end{array}$ & Prime Minister's \\
\hline 9. & $\begin{array}{l}\text { Department of Waqf, Zakat and Hajj } \\
\text { (JAWHAR) }\end{array}$ & Prime Minister's \\
\hline 10. & $\begin{array}{l}\text { Division of Ceremonies and } \\
\text { International Conferences Secretariat } \\
\text { (BIUPA) }\end{array}$ & Prime Minister's \\
\hline 11. & $\begin{array}{l}\text { Development of Hawkers, Petty } \\
\text { Traders and Hindu Endowment Unit } \\
\text { (UPPPKWH) }\end{array}$ & Prime Minister's \\
\hline 12. & $\begin{array}{l}\text { Implementation Coordination Unit } \\
\text { (ICU) }\end{array}$ & Prime Minister's \\
\hline 13. & $\begin{array}{lrr}\text { Integrity } & \text { and } & \text { Governance } \\
\text { Department (JITN) } & \end{array}$ & Prime Minister's \\
\hline 14. & Legal Affairs Division (BHEUU) & Prime Minister's \\
\hline
\end{tabular}


INTERNATIONAL JOURNAL OF ACADEMIC RESEARCH IN BUSINESS AND SOCIAL SCIENCES

Vol. 9, No. 7, July, 2019, E-ISSN: 2222-6990 @ 2019 HRMARS

\begin{tabular}{|c|c|c|}
\hline & $\begin{array}{l}\text { Legal Aid Department (JBG) } \\
\text { Malaysian Department of } \\
\text { Insolvencies (Mdl) }\end{array}$ & \\
\hline 15. & $\begin{array}{l}\text { Legal and Judicial Training Institute } \\
\text { (ILKAP) }\end{array}$ & Prime Minister's \\
\hline 16. & $\begin{array}{l}\text { Malaysian Islamic Development } \\
\text { Department (JAKIM) } \\
\text { Federal Territories' Islamic Affairs } \\
\text { Department (JAWI) }\end{array}$ & Prime Minister's \\
\hline 17. & $\begin{array}{l}\text { Malaysian Industry-Government } \\
\text { Group for High Technology (MiGHT) }\end{array}$ & Prime Minister's \\
\hline 18. & $\begin{array}{l}\text { Malaysian Maritime Enforcement } \\
\text { Affairs Division (BHEPMM) }\end{array}$ & Prime Minister's \\
\hline 19. & $\begin{array}{l}\text { Malaysian Sharia } \quad \text { Judiciary } \\
\text { Department (JKSM) } \\
\text { Federal Territories' Sharia Court } \\
\text { (MSWP) }\end{array}$ & Prime Minister's \\
\hline 20. & $\begin{array}{l}\text { National Governance, Integrity and } \\
\text { Anti-Corruption Centre (GIACC) }\end{array}$ & Prime Minister's \\
\hline 21. & National Security Council (MKN) & Prime Minister's \\
\hline 22. & $\begin{array}{l}\text { Office of Sabah Federal Secretary } \\
\text { (PSUP Sabah) }\end{array}$ & Prime Minister's \\
\hline 23. & $\begin{array}{l}\text { Office of Sarawak Federal Secretary } \\
\text { (PSUP Sarawak) }\end{array}$ & Prime Minister's \\
\hline 24. & $\begin{array}{l}\text { Office of the Chief Government } \\
\text { Security Officer (CGSO) }\end{array}$ & Prime Minister's \\
\hline
\end{tabular}

1221 


\begin{tabular}{|l|l|l|}
\hline 25. & $\begin{array}{l}\text { Office of the Chief Registrar of the } \\
\text { Federal Court [3] (PKPMP) }\end{array}$ & Prime Minister's \\
\hline 26. & $\begin{array}{l}\text { Office of the Director of Land and } \\
\text { Mines of Federal Territories (PTGWP) } \\
\text { Office of Land and Mines of Labuan } \\
\text { Office of Land and Mines of Putrajaya }\end{array}$ & Prime Minister's \\
\hline 27. & $\begin{array}{l}\text { Office of the Former Prime Minister, } \\
\text { Tun Abdullah Ahmad Badawi }\end{array}$ & Prime Minister's \\
\hline 28. & $\begin{array}{l}\text { Office of the Former Prime Minister, } \\
\text { Dato' Sri Mohd. Najib Abdul Razak }\end{array}$ & Prime Minister's \\
\hline 29. & $\begin{array}{l}\text { Office of the Keeper of the Rulers' } \\
\text { Seal (Website) (PMBRR) }\end{array}$ & Prime Minister's \\
\hline 30. & $\begin{array}{l}\text { Office of the Mufti of Federal } \\
\text { Territories (PMWP) }\end{array}$ & Prime Minister's \\
\hline 31. & $\begin{array}{l}\text { Performance and Delivery } \\
\text { Management Unit (PEMANDU) }\end{array}$ & Prime Minister's \\
\hline 32. & Property Management Division (BPH) & Prime Minister's \\
\hline 33. & Protection Division & Prime Minister's \\
\hline 34. & Public Complaints Bureau (BPA/PCB) & Prime Minister's \\
\hline 35. & $\begin{array}{l}\text { Public Service Department (JPA) } \\
\text { Malaysian Institute of Public } \\
\text { Administration (INTAN) }\end{array}$ & Prime Minister's \\
\hline 36. & Research Division & Prime Minister's \\
\hline 37. & Secretariat of Federal & Prime Minister's \\
\hline 38. & $\begin{array}{l}\text { SME and Microcredit Development } \\
\text { Unit }\end{array}$ & Prime Minister's \\
\hline 39. & Strategic Special Unit (UKS) & Prime Minister's \\
\hline 40. & Territories Earthworks Committee \\
\hline 41. & $\begin{array}{l}\text { Enforcement Agency Integrity } \\
\text { Commission (SIAP/EAIC) }\end{array}$ & Prime Minister's \\
\hline
\end{tabular}


INTERNATIONAL JOURNAL OF ACADEMIC RESEARCH IN BUSINESS AND SOCIAL SCIENCES Vol. 9, No. 7, July, 2019, E-ISSN: 2222-6990 @ 2019 HRMARS

\begin{tabular}{|c|c|c|}
\hline 42. & $\begin{array}{l}\text { Federal Territories' Islamic Affairs } \\
\text { Council (MAIWP) } \\
\text { MAIWP Zakat Collection Centre (PPZ- } \\
\text { MAIWP) }\end{array}$ & Prime Minister's \\
\hline 43. & National Innovation Agency* (AIM) & Prime Minister's \\
\hline 44. & Pilgrims Fund Board $(\mathrm{TH})$ & Prime Minister's \\
\hline 45. & $\begin{array}{l}\text { Rulers' and Governors' Higher } \\
\text { Education Scholarship Fund }\end{array}$ & Prime Minister's \\
\hline 46. & $\begin{array}{l}\text { Alhijrah Media Corporation (TV } \\
\text { Alhijrah) }\end{array}$ & Prime Minister's \\
\hline 47. & $\begin{array}{l}\text { Institute for Strategic and } \\
\text { International Studies (ISIS) }\end{array}$ & Prime Minister's \\
\hline 48. & $\begin{array}{l}\text { International Multilateral Partnership } \\
\text { Against Cyber Threats (IMPACT) }\end{array}$ & Prime Minister's \\
\hline 49. & $\begin{array}{lll}\text { Malaysian } & \text { Integrity } & \text { Institute } \\
\text { (INTEGRITI) } & & \\
\end{array}$ & Prime Minister's \\
\hline 50. & $\begin{array}{l}\text { Malaysian Islamic Understanding } \\
\text { Institute (IKIM) }\end{array}$ & Prime Minister's \\
\hline 51. & $\begin{array}{lll}\begin{array}{l}\text { Petroliam } \\
\text { (PETRONAS) }\end{array} & \text { Nasional } & \text { Berhad } \\
\end{array}$ & Prime Minister's \\
\hline 52. & $\begin{array}{l}\text { Islamic Dakwah Foundation of } \\
\text { Malaysia (YADIM) }\end{array}$ & Prime Minister's \\
\hline 53. & $\begin{array}{l}\text { Islamic Economic Development } \\
\text { Foundation of Malaysia (YaPEIM) }\end{array}$ & Prime Minister's \\
\hline 54. & $\begin{array}{l}\text { Waqaf Foundation of Malaysia } \\
\text { (YWM) }\end{array}$ & Prime Minister's \\
\hline 55. & $\begin{array}{l}\text { Kuala Lumpur Regional Centre for } \\
\text { Arbitration (KLRCA) }\end{array}$ & Prime Minister's \\
\hline 56. & $\begin{array}{l}\text { Malaysian Agricultural Research and } \\
\text { Development Institute (MARDI) }\end{array}$ & $\begin{array}{l}\text { Ministry of Agriculture } \\
\text { and Agro-based Industry }\end{array}$ \\
\hline
\end{tabular}


INTERNATIONAL JOURNAL OF ACADEMIC RESEARCH IN BUSINESS AND SOCIAL SCIENCES Vol. 9, No. 7, July, 2019, E-ISSN: 2222-6990 @ 2019 HRMARS

\begin{tabular}{|l|l|l|}
\hline 57. & $\begin{array}{l}\text { Farmers' Organization Authority* } \\
\text { (LPP) }\end{array}$ & $\begin{array}{l}\text { Ministry of Agriculture } \\
\text { and Agro-based Industry }\end{array}$ \\
\hline 58. & $\begin{array}{l}\text { Federal Agricultural Marketing } \\
\text { Authority* (FAMA) }\end{array}$ & $\begin{array}{l}\text { Ministry of Agriculture } \\
\text { and Agro-based Industry }\end{array}$ \\
\hline 59. & $\begin{array}{l}\text { Malaysian Fisheries Development } \\
\text { Authority* (LKIM) }\end{array}$ & $\begin{array}{l}\text { Ministry of Agriculture } \\
\text { and Agro-based Industry }\end{array}$ \\
\hline 60. & $\begin{array}{l}\text { Muda Agricultural Development } \\
\text { Authority* (MADA) }\end{array}$ & $\begin{array}{l}\text { Ministry of Agriculture } \\
\text { and Agro-based Industry }\end{array}$ \\
\hline 61. & $\begin{array}{l}\text { Malaysian Pineapple Industrial } \\
\text { Authority* (MPIB) }\end{array}$ & $\begin{array}{l}\text { Ministry of Agriculture } \\
\text { and Agro-based Industry }\end{array}$ \\
\hline 62. & $\begin{array}{l}\text { Kemubu Agricultural Development } \\
\text { Authority* (KADA) }\end{array}$ & $\begin{array}{l}\text { Ministry of Agriculture } \\
\text { and Agro-based Industry }\end{array}$ \\
\hline 63. & $\begin{array}{l}\text { Department of Agriculture (DOA) } \\
\text { 6inistry of Agriculture } \\
\text { and Agro-based Industry }\end{array}$ \\
\hline 64. & $\begin{array}{l}\text { Department of Veterinary Service } \\
\text { (DVS) }\end{array}$ & $\begin{array}{l}\text { Ministry of Agriculture } \\
\text { and Agro-based Industry }\end{array}$ \\
\hline 65. & Department of Fisheries (DOF) & $\begin{array}{l}\text { Ministry of Agriculture } \\
\text { and Agro-based Industry }\end{array}$ \\
\hline 66. & $\begin{array}{l}\text { Malaysian Quarantine and Inspection } \\
\text { Services (MAQIS) }\end{array}$ & $\begin{array}{l}\text { Ministry of Agriculture } \\
\text { and Agro-based Industry }\end{array}$ \\
\hline 67. & Agrobank & $\begin{array}{l}\text { Ministry of Agriculture } \\
\text { and Agro-based Industry }\end{array}$ \\
\hline 68. & CyberSecurity Malaysia & $\begin{array}{l}\text { Ministry an } \\
\text { Communication and } \\
\text { Multimedia }\end{array}$ \\
\hline 69. & Department of Broadcasting (RTM) & $\begin{array}{l}\text { Ministry } \\
\text { Communication and } \\
\text { Multimedia }\end{array}$ \\
\hline
\end{tabular}


INTERNATIONAL JOURNAL OF ACADEMIC RESEARCH IN BUSINESS AND SOCIAL SCIENCES

Vol. 9, No. 7, July, 2019, E-ISSN: 2222-6990 @ 2019 HRMARS

\begin{tabular}{|c|c|c|}
\hline 70. & Department of Information (JAPEN) & $\begin{array}{l}\text { Ministry } \\
\text { Communication } \\
\text { Multimedia }\end{array}$ \\
\hline 71. & $\begin{array}{l}\text { Department of Personal Data } \\
\text { Protection (JPDP) }\end{array}$ & $\begin{array}{l}\text { Ministry } \\
\text { Communication } \\
\text { Multimedia }\end{array}$ \\
\hline 72. & 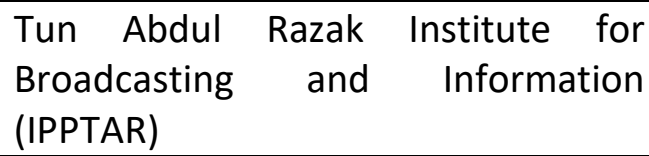 & $\begin{array}{l}\text { Ministry } \\
\text { Communication } \\
\text { Multimedia }\end{array}$ \\
\hline 73. & $\begin{array}{l}\text { Malaysian Administrative } \\
\text { Modernisation and Management } \\
\text { Planning Unit (MAMPU) }\end{array}$ & $\begin{array}{l}\text { Ministry } \\
\text { Communication } \\
\text { Multimedia }\end{array}$ \\
\hline 74. & $\begin{array}{l}\text { Malaysian Communication and } \\
\text { Multimedia Commission (SKMM) }\end{array}$ & $\begin{array}{l}\text { Ministry } \\
\text { Communication } \\
\text { Multimedia }\end{array}$ \\
\hline 75. & $\begin{array}{lrl}\text { Malaysian } & \text { Digital } & \text { Economy } \\
\text { Corporation }(\mathrm{MDEC}) & \end{array}$ & $\begin{array}{l}\text { Ministry } \\
\text { Communication } \\
\text { Multimedia }\end{array}$ \\
\hline 76. & $\begin{array}{l}\text { Malaysian National News Agency } \\
\text { (BERNAMA) }\end{array}$ & $\begin{array}{l}\text { Ministry } \\
\text { Communication } \\
\text { Multimedia }\end{array}$ \\
\hline 77. & $\begin{array}{l}\text { National } \quad \text { Film } \\
\text { Corporation (FINAS) }\end{array}$ & $\begin{array}{l}\text { Ministry } \\
\text { Communication } \\
\text { Multimedia }\end{array}$ \\
\hline 78. & MyNIC & $\begin{array}{l}\text { Ministry } \\
\text { Communication } \\
\text { Multimedia }\end{array}$ \\
\hline 79. & Malaysian Armed Forces (ATM) & Ministry of Defense \\
\hline 80. & $\begin{array}{l}\text { Armed Forces Retirees' } \\
\text { Corporation (PERHEBAT) }\end{array}$ & Ministry of Defense \\
\hline
\end{tabular}


INTERNATIONAL JOURNAL OF ACADEMIC RESEARCH IN BUSINESS AND SOCIAL SCIENCES Vol. 9, No. 7, July, 2019, E-ISSN: 2222-6990 @ 2019 HRMARS

\begin{tabular}{|c|c|c|}
\hline 81. & $\begin{array}{l}\text { ATM Veteran Affairs Department } \\
\text { (JHEV) }\end{array}$ & Ministry of Defense \\
\hline 82. & $\begin{array}{l}\text { Malaysian Institute of Defense and } \\
\text { Security (MIDAS) }\end{array}$ & Ministry of Defense \\
\hline 83. & $\begin{array}{l}\text { Science and Technology Research } \\
\text { Institute for Defense (STRIDE) }\end{array}$ & Ministry of Defense \\
\hline 84. & Companies Commission (SSM) & $\begin{array}{l}\text { Ministry of Domestic } \\
\text { Trade and Consumer } \\
\text { Affairs }\end{array}$ \\
\hline 85. & Competition Commission (MyCC) & $\begin{array}{l}\text { Ministry of Domestic } \\
\text { Trade and Consumer } \\
\text { Affairs }\end{array}$ \\
\hline 86. & $\begin{array}{l}\text { Malaysian Intellectual Property } \\
\text { Corporation (MyIPO) }\end{array}$ & $\begin{array}{l}\text { Ministry of Domestic } \\
\text { Trade and Consumer } \\
\text { Affairs }\end{array}$ \\
\hline 87. & Perbadanan Nasional Berhad (PNS) & $\begin{array}{l}\text { Ministry of Domestic } \\
\text { Trade and Consumer } \\
\text { Affairs }\end{array}$ \\
\hline 88. & Department of Statistics (DOSM) & $\begin{array}{l}\text { Ministry of Economic } \\
\text { Affairs }\end{array}$ \\
\hline 89. & Economic Planning Unit (EPU) & $\begin{array}{l}\text { Ministry of Economic } \\
\text { Affairs }\end{array}$ \\
\hline 90. & FELDA Outreach Unit (Seranta) & $\begin{array}{l}\text { Ministry of Economic } \\
\text { Affairs }\end{array}$ \\
\hline 91. & FELDA Regulatory Unit (UKSF) & $\begin{array}{l}\text { Ministry of Economic } \\
\text { Affairs }\end{array}$ \\
\hline 92. & $\begin{array}{l}\text { Public-Private Partnership Unit } \\
\text { (UKAS) }\end{array}$ & $\begin{array}{l}\text { Ministry of Economic } \\
\text { Affairs }\end{array}$ \\
\hline 93. & $\begin{array}{l}\text { Federal Land Development Authority } \\
\text { (FELDA) }\end{array}$ & $\begin{array}{l}\text { Ministry of Economic } \\
\text { Affairs }\end{array}$ \\
\hline
\end{tabular}


INTERNATIONAL JOURNAL OF ACADEMIC RESEARCH IN BUSINESS AND SOCIAL SCIENCES Vol. 9, No. 7, July, 2019, E-ISSN: 2222-6990 @ 2019 HRMARS

\begin{tabular}{|c|c|c|}
\hline 94. & $\begin{array}{l}\text { Langkawi Development Authority } \\
\text { (LADA) }\end{array}$ & $\begin{array}{l}\text { Ministry of Economic } \\
\text { Affairs }\end{array}$ \\
\hline 95. & Amanah Raya Berhad (ARB) & $\begin{array}{l}\text { Ministry of Economic } \\
\text { Affairs }\end{array}$ \\
\hline 96. & $\begin{array}{l}\text { Bumiputera Agenda Steering Unit } \\
\text { (TERAJU) }\end{array}$ & $\begin{array}{l}\text { Ministry of Economic } \\
\text { Affairs }\end{array}$ \\
\hline 97. & $\begin{array}{l}\text { Bumiputera Education Steering } \\
\text { Foundation (YPPB) }\end{array}$ & $\begin{array}{l}\text { Ministry of Economic } \\
\text { Affairs }\end{array}$ \\
\hline 98. & Ekuiti Nasional Berhad (EKUINAS) & $\begin{array}{l}\text { Ministry of Economic } \\
\text { Affairs }\end{array}$ \\
\hline 99. & Ekuiti Nasional Foundation (YEN) & $\begin{array}{l}\text { Ministry of Economic } \\
\text { Affairs }\end{array}$ \\
\hline 100. & $\begin{array}{l}\text { Federal Land Combination and } \\
\text { Recovery Authority (FELCRA) }\end{array}$ & $\begin{array}{l}\text { Ministry of Economic } \\
\text { Affairs }\end{array}$ \\
\hline 101. & $\begin{array}{lrr}\text { Halal Industry } & \text { Development } \\
\text { Corporation (HDC) } & \\
\end{array}$ & $\begin{array}{l}\text { Ministry of Economic } \\
\text { Affairs }\end{array}$ \\
\hline 102. & $\begin{array}{ll}\text { Malaysia } & \text { Petroleum } \\
\text { Corporation (MPRC) } & \text { Resources } \\
\end{array}$ & $\begin{array}{l}\text { Ministry of Economic } \\
\text { Affairs }\end{array}$ \\
\hline 103. & Dewan Bahasa dan Pustaka* (DBP) & Ministry of Education \\
\hline 104. & Higher Education Department (JPT) & Ministry of Education \\
\hline 105. & $\begin{array}{l}\text { Malaysian Examination Council* } \\
\text { (MPM) }\end{array}$ & Ministry of Education \\
\hline 106. & $\begin{array}{l}\text { Malaysian Institute of Translation \& } \\
\text { Books (ITBM) }\end{array}$ & Ministry of Education \\
\hline 107. & Malaysian National Library (PNM) & Ministry of Education \\
\hline 108. & $\begin{array}{l}\text { Malaysian Qualification Agency* } \\
\text { (MQA) }\end{array}$ & Ministry of Education \\
\hline 109. & $\begin{array}{l}\text { National Higher Education Fund } \\
\text { Corporation (PTPTN) }\end{array}$ & Ministry of Education \\
\hline
\end{tabular}


INTERNATIONAL JOURNAL OF ACADEMIC RESEARCH IN BUSINESS AND SOCIAL SCIENCES Vol. 9, No. 7, July, 2019, E-ISSN: 2222-6990 @ 2019 HRMARS

\begin{tabular}{|c|c|c|}
\hline 110. & PADU Corporation & Ministry of Education \\
\hline 111. & $\begin{array}{l}\text { Polytechnic and Community College } \\
\text { Education Department (JPPKK) }\end{array}$ & Ministry of Education \\
\hline 112. & National Archives of Malaysia (ANM) & Ministry of Education \\
\hline 113. & $\begin{array}{l}\text { Atom Energy Licensing Board } \\
\text { (LPTA/AELB) }\end{array}$ & $\begin{array}{l}\text { Ministry of Energy, } \\
\text { Technology, Science, } \\
\text { Climate Change and } \\
\text { Environment }\end{array}$ \\
\hline 114. & Department of Biosafety (JBK) & $\begin{array}{l}\text { Ministry of Energy, } \\
\text { Technology, Science, } \\
\text { Climate Change and } \\
\text { Environment }\end{array}$ \\
\hline 115. & $\begin{array}{l}\text { Department of Environment } \\
\text { (JAS/DOE) }\end{array}$ & $\begin{array}{l}\text { Ministry of Energy, } \\
\text { Technology, Science, } \\
\text { Climate Change and } \\
\text { Environment }\end{array}$ \\
\hline 116. & $\begin{array}{l}\text { Malaysian Chemistry Department } \\
\text { (JKM) }\end{array}$ & $\begin{array}{l}\text { Ministry of Energy, } \\
\text { Technology, Science, } \\
\text { Climate Change and } \\
\text { Environment }\end{array}$ \\
\hline 117. & $\begin{array}{lr}\text { Malaysian } & \text { Meteorological } \\
\text { Department } & \text { (MMD/MET Malaysia) } \\
\text { (Website) } & \end{array}$ & $\begin{array}{l}\text { Ministry of Energy, } \\
\text { Technology, Science, } \\
\text { Climate Change and } \\
\text { Environment }\end{array}$ \\
\hline 118. & $\begin{array}{l}\text { Malaysian Nuclear Agency (Nuclear } \\
\text { Malaysia) (Website) }\end{array}$ & $\begin{array}{l}\text { Ministry of Energy, } \\
\text { Technology, Science, } \\
\text { Climate Change and } \\
\text { Environment }\end{array}$ \\
\hline 119. & $\begin{array}{l}\text { Malaysian Remote Sensing Agency } \\
\text { (MRSA) }\end{array}$ & $\begin{array}{lr}\text { Ministry of } & \text { Energy, } \\
\text { Technology, } & \text { Science, } \\
\end{array}$ \\
\hline
\end{tabular}


INTERNATIONAL JOURNAL OF ACADEMIC RESEARCH IN BUSINESS AND SOCIAL SCIENCES Vol. 9, No. 7, July, 2019, E-ISSN: 2222-6990 @ 2019 HRMARS

\begin{tabular}{|c|c|c|}
\hline & & $\begin{array}{l}\text { Climate Change and } \\
\text { Environment }\end{array}$ \\
\hline 120. & $\begin{array}{l}\text { Malaysian Standards Department } \\
\text { (JSM/Standards Malaysia) }\end{array}$ & $\begin{array}{l}\text { Ministry of Energy, } \\
\text { Technology, Science, } \\
\text { Climate Change and } \\
\text { Environment }\end{array}$ \\
\hline 121. & $\begin{array}{l}\text { Malaysian Sustainable Energy } \\
\text { Development Authority (SEDA) }\end{array}$ & $\begin{array}{l}\text { Ministry of Energy, } \\
\text { Technology, Science, } \\
\text { Climate Change and } \\
\text { Environment }\end{array}$ \\
\hline 122. & National Science Centre (PSN) & $\begin{array}{l}\text { Ministry of Energy, } \\
\text { Technology, Science, } \\
\text { Climate Change and } \\
\text { Environment }\end{array}$ \\
\hline 123. & $\begin{array}{l}\text { National Space Agency (ANGKASA) } \\
\text { (Website) }\end{array}$ & $\begin{array}{l}\text { Ministry of Energy, } \\
\text { Technology, Science, } \\
\text { Climate Change and } \\
\text { Environment }\end{array}$ \\
\hline 124. & Academy of Science, Malaysia (ASM) & $\begin{array}{l}\text { Ministry of Energy, } \\
\text { Technology, Science, } \\
\text { Climate Change and } \\
\text { Environment }\end{array}$ \\
\hline 125. & Energy Commission (ST) (Website) & $\begin{array}{l}\text { Ministry of Energy, } \\
\text { Technology, Science, } \\
\text { Climate Change and } \\
\text { Environment }\end{array}$ \\
\hline 126. & $\begin{array}{l}\text { Malaysian Board of Technologists } \\
\text { (MBOT) }\end{array}$ & $\begin{array}{l}\text { Ministry of Energy, } \\
\text { Technology, Science, } \\
\text { Climate Change and } \\
\text { Environment }\end{array}$ \\
\hline
\end{tabular}


INTERNATIONAL JOURNAL OF ACADEMIC RESEARCH IN BUSINESS AND SOCIAL SCIENCES Vol. 9, No. 7, July, 2019, E-ISSN: 2222-6990 @ 2019 HRMARS

\begin{tabular}{|c|c|c|}
\hline 127. & $\begin{array}{l}\text { Malaysian Astronauts Foundation } \\
\text { (YAM) }\end{array}$ & $\begin{array}{l}\text { Ministry of Energy, } \\
\text { Technology, Science, } \\
\text { Climate Change and } \\
\text { Environment }\end{array}$ \\
\hline 128. & $\begin{array}{l}\text { Malaysian Foundation for Innovation } \\
\text { (YIM) }\end{array}$ & $\begin{array}{l}\text { Ministry of Energy, } \\
\text { Technology, Science, } \\
\text { Climate Change and } \\
\text { Environment }\end{array}$ \\
\hline 129. & $\begin{array}{l}\text { Malaysian Institute of Chemistry } \\
\text { (IKM) }\end{array}$ & $\begin{array}{l}\text { Ministry of Energy, } \\
\text { Technology, Science, } \\
\text { Climate Change and } \\
\text { Environment }\end{array}$ \\
\hline 130. & $\begin{array}{lll}\text { Sultan Mizan } & \text { Foundation for } \\
\text { Antarctic } & \text { Research (YPASM) } & \end{array}$ & $\begin{array}{l}\text { Ministry of Energy, } \\
\text { Technology, Science, } \\
\text { Climate Change and } \\
\text { Environment }\end{array}$ \\
\hline 131. & $\begin{array}{l}\text { Astronautic Technology Sdn. Bhd. } \\
\text { (ATSB) }\end{array}$ & $\begin{array}{l}\text { Ministry of Energy, } \\
\text { Technology, Science, } \\
\text { Climate Change and } \\
\text { Environment }\end{array}$ \\
\hline 132. & Bioeconomy Corporation & $\begin{array}{l}\text { Ministry of Energy, } \\
\text { Technology, Science, } \\
\text { Climate Change and } \\
\text { Environment }\end{array}$ \\
\hline 133. & Inno Biologics Sdn. Bhd. & $\begin{array}{l}\text { Ministry of Energy, } \\
\text { Technology, Science, } \\
\text { Climate Change and } \\
\text { Environment }\end{array}$ \\
\hline 134. & Kumpulan Modal Perdana Sdn. Bhd. & $\begin{array}{lr}\text { Ministry of } & \text { Energy, } \\
\text { Technology, } & \text { Science, }\end{array}$ \\
\hline
\end{tabular}


INTERNATIONAL JOURNAL OF ACADEMIC RESEARCH IN BUSINESS AND SOCIAL SCIENCES Vol. 9, No. 7, July, 2019, E-ISSN: 2222-6990@ 2019 HRMARS

\begin{tabular}{|c|c|c|}
\hline & & $\begin{array}{l}\text { Climate Change and } \\
\text { Environment }\end{array}$ \\
\hline 135. & $\begin{array}{l}\text { Malaysia Debt Ventures Berhad } \\
\text { (MDV) }\end{array}$ & $\begin{array}{l}\text { Ministry of Energy, } \\
\text { Technology, Science, } \\
\text { Climate Change and } \\
\text { Environment }\end{array}$ \\
\hline 136. & Malaysia Design Council (MRM) & $\begin{array}{l}\text { Ministry of Energy, } \\
\text { Technology, Science, } \\
\text { Climate Change and } \\
\text { Environment }\end{array}$ \\
\hline 137. & $\begin{array}{l}\text { Malaysia } \quad \text { Green } \\
\text { Corporation (GreenTech Malaysia) }\end{array}$ & $\begin{array}{l}\text { Ministry of Energy, } \\
\text { Technology, Science, } \\
\text { Climate Change and } \\
\text { Environment }\end{array}$ \\
\hline 138. & $\begin{array}{l}\text { Malaysian Nuclear } \\
\text { Corporation (MNPC) }\end{array}$ & $\begin{array}{l}\text { Ministry of Energy, } \\
\text { Technology, Science, } \\
\text { Climate Change and } \\
\text { Environment }\end{array}$ \\
\hline 139. & Malaysia Venture Capital (MAVCAP) & $\begin{array}{l}\text { Ministry of Energy, } \\
\text { Technology, Science, } \\
\text { Climate Change and } \\
\text { Environment }\end{array}$ \\
\hline 140. & $\begin{array}{l}\text { Malaysian Technology Development } \\
\text { Corporation (MTDC) }\end{array}$ & $\begin{array}{l}\text { Ministry of Energy, } \\
\text { Technology, Science, } \\
\text { Climate Change and } \\
\text { Environment }\end{array}$ \\
\hline 141. & $\begin{array}{l}\text { National Institutes of Biotechnology } \\
\text { Malaysia (NIBM) }\end{array}$ & $\begin{array}{l}\text { Ministry of Energy, } \\
\text { Technology, Science, } \\
\text { Climate Change and } \\
\text { Environment }\end{array}$ \\
\hline
\end{tabular}


INTERNATIONAL JOURNAL OF ACADEMIC RESEARCH IN BUSINESS AND SOCIAL SCIENCES Vol. 9, No. 7, July, 2019, E-ISSN: 2222-6990 @ 2019 HRMARS

\begin{tabular}{|c|c|c|}
\hline 142. & MIMOS Berhad (Website) & $\begin{array}{l}\text { Ministry of Energy, } \\
\text { Technology, Science, } \\
\text { Climate Change and } \\
\text { Environment }\end{array}$ \\
\hline 143. & NanoMalaysia Berhad & $\begin{array}{l}\text { Ministry of Energy, } \\
\text { Technology, Science, } \\
\text { Climate Change and } \\
\text { Environment }\end{array}$ \\
\hline 144. & SIRIM Berhad (Website) & $\begin{array}{l}\text { Ministry of Energy, } \\
\text { Technology, Science, } \\
\text { Climate Change and } \\
\text { Environment }\end{array}$ \\
\hline 145. & 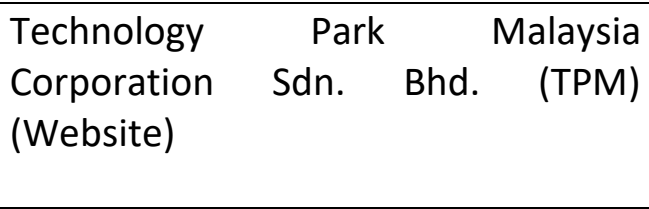 & $\begin{array}{l}\text { Ministry of Energy, } \\
\text { Technology, Science, } \\
\text { Climate Change and } \\
\text { Environment }\end{array}$ \\
\hline 146. & Yayasan Hijau (yaHijau) & $\begin{array}{l}\text { Ministry of Energy, } \\
\text { Technology, Science, } \\
\text { Climate Change and } \\
\text { Environment }\end{array}$ \\
\hline 147. & Co-operatives Commission (SKM) & $\begin{array}{ll}\text { Ministry of } \\
\text { Entrepreneurship } \\
\text { Development }\end{array}$ \\
\hline 148. & $\begin{array}{l}\text { Malaysian } \text { Co-operative } \text { College } \\
\text { (MKM) }\end{array}$ & $\begin{array}{l}\text { Ministry } \\
\text { Entrepreneurship } \\
\text { Development }\end{array}$ \\
\hline 149. & $\begin{array}{l}\text { SME Corporation Malaysia* (SME } \\
\text { Corp) }\end{array}$ & $\begin{array}{l}\text { Ministry } \\
\text { Entrepreneurship } \\
\text { Development }\end{array}$ \\
\hline
\end{tabular}


INTERNATIONAL JOURNAL OF ACADEMIC RESEARCH IN BUSINESS AND SOCIAL SCIENCES

Vol. 9, No. 7, July, 2019, E-ISSN: 2222-6990 @ 2019 HRMARS

\begin{tabular}{|c|c|c|}
\hline 150. & $\begin{array}{l}\text { Small and Medium Enterprise Bank } \\
\text { (SME Bank) }\end{array}$ & $\begin{array}{l}\text { Ministry } \\
\text { Entrepreneurship } \\
\text { Development }\end{array}$ \\
\hline 151. & TEKUN Nasional & $\begin{array}{l}\text { Ministry } \\
\text { Entrepreneurship } \\
\text { Development }\end{array}$ \\
\hline 152. & $\begin{array}{l}\text { Perbadanan Usahawan Nasional } \\
\text { Berhad (PUNB) }\end{array}$ & $\begin{array}{l}\text { Ministry } \\
\text { Entrepreneurship } \\
\text { Development }\end{array}$ \\
\hline 153. & Uda Holdings Berhad & $\begin{array}{l}\text { Ministry } \\
\text { Entrepreneurship } \\
\text { Development }\end{array}$ \\
\hline 154. & $\begin{array}{l}\text { Bank Kerjasama Rakyat Malaysia } \\
\text { Berhad (Bank Rakyat) }\end{array}$ & $\begin{array}{l}\text { Ministry } \\
\text { Entrepreneurship } \\
\text { Development }\end{array}$ \\
\hline 155. & $\begin{array}{lll}\text { Institut } & \text { Keusahawanan } & \text { Negara } \\
\text { (INSKEN) } & & \end{array}$ & $\begin{array}{l}\text { Ministry } \\
\text { Entrepreneurship } \\
\text { Development }\end{array}$ \\
\hline 156. & $\begin{array}{l}\text { Professional Training \& Education For } \\
\text { Growing Entrepreneurs (Protege) }\end{array}$ & $\begin{array}{l}\text { Ministry } \\
\text { Entrepreneurship } \\
\text { Development }\end{array}$ \\
\hline 157. & $\begin{array}{l}\text { Malaysian Global Innovation \& } \\
\text { Creativity Centre (MAGIC) }\end{array}$ & $\begin{array}{l}\text { Ministry } \\
\text { Entrepreneurship } \\
\text { Development }\end{array}$ \\
\hline 158. & $\begin{array}{l}\text { Kuala Lumpur City Hall (DBKL) } \\
\text { (Website) }\end{array}$ & $\begin{array}{l}\text { Ministry of Federal } \\
\text { Territories }\end{array}$ \\
\hline 159. & $\begin{array}{l}\text { Kampong Bharu } \quad \text { Development } \\
\text { Corporation (PKB) }\end{array}$ & $\begin{array}{l}\text { Ministry of } \\
\text { Territories }\end{array}$ \\
\hline 160. & Labuan Corporation (PL) & $\begin{array}{l}\text { Ministry of Federal } \\
\text { Territories }\end{array}$ \\
\hline
\end{tabular}


INTERNATIONAL JOURNAL OF ACADEMIC RESEARCH IN BUSINESS AND SOCIAL SCIENCES Vol. 9, No. 7, July, 2019, E-ISSN: 2222-6990 @ 2019 HRMARS

\begin{tabular}{|c|c|c|}
\hline 161. & Putrajaya Corporation (PPj) & $\begin{array}{l}\text { Ministry of Federal } \\
\text { Territories }\end{array}$ \\
\hline 162. & $\begin{array}{l}\text { Federal Territories' Sports Council } \\
\text { (MSWP) }\end{array}$ & $\begin{array}{l}\text { Ministry of Federal } \\
\text { Territories }\end{array}$ \\
\hline 163. & $\begin{array}{l}\text { Accountant-General's Department } \\
\text { (ANM) }\end{array}$ & Ministry of Finance \\
\hline 164. & $\begin{array}{l}\text { Property Assessment and Services } \\
\text { Department (JPPH) }\end{array}$ & Ministry of Finance \\
\hline 165. & $\begin{array}{lll}\text { Royal Malaysian } & \text { Customs } \\
\text { Department (JKDM) } & \end{array}$ & Ministry of Finance \\
\hline 166. & Bank Simpanan Nasional (BSN) & Ministry of Finance \\
\hline 167. & Central Bank of Malaysia (BNM) & Ministry of Finance \\
\hline 168. & $\begin{array}{l}\text { East Coast Economic Region } \\
\text { Development Council (ECER DC) }\end{array}$ & Ministry of Finance \\
\hline 169. & $\begin{array}{lll}\begin{array}{l}\text { Employees' } \\
\text { (KWSP/EPF) }\end{array} & \text { Provident } & \text { Fund* } \\
\end{array}$ & Ministry of Finance \\
\hline 170. & Inland Revenue Board (LHDN) & Ministry of Finance \\
\hline 171. & $\begin{array}{lll}\text { Iskandar Region } & \text { Development } \\
\text { Authority (IRDA) } & \\
\end{array}$ & Ministry of Finance \\
\hline 172. & Khazanah Nasional Berhad & Ministry of Finance \\
\hline 173. & $\begin{array}{l}\text { Labuan Financial Services Authority* } \\
\text { (Labuan FSA) }\end{array}$ & Ministry of Finance \\
\hline 174. & Malaysian Totalisator Board & Ministry of Finance \\
\hline 175. & $\begin{array}{l}\text { Northern Corridor Implementation } \\
\text { Authority }(\mathrm{NClA})\end{array}$ & Ministry of Finance \\
\hline 176. & $\begin{array}{l}\text { Public Sector Housing Financing } \\
\text { Authority* (LPPSA) }\end{array}$ & Ministry of Finance \\
\hline 177. & $\begin{array}{l}\text { Regional Corridor Development } \\
\text { Authority (RECODA) }\end{array}$ & Ministry of Finance \\
\hline
\end{tabular}


INTERNATIONAL JOURNAL OF ACADEMIC RESEARCH IN BUSINESS AND SOCIAL SCIENCES Vol. 9, No. 7, July, 2019, E-ISSN: 2222-6990 @ 2019 HRMARS

\begin{tabular}{|c|c|c|}
\hline 178. & $\begin{array}{l}\text { Retirement Fund (Incorporated) } \\
\text { (KWAP) }\end{array}$ & Ministry of Finance \\
\hline 179. & $\begin{array}{l}\text { Sabah Economic Development and } \\
\text { Investment Authority (SEDIA) }\end{array}$ & Ministry of Finance \\
\hline 180. & Securities Commission (SC) & Ministry of Finance \\
\hline 181. & Bursa Malaysia Berhad & Ministry of Finance \\
\hline 182. & Yayasan Tun Razak (YTR) & Ministry of Finance \\
\hline 183. & ASEAN-Malaysia National Secretariat & $\begin{array}{l}\text { Ministry of Foreign } \\
\text { Affairs }\end{array}$ \\
\hline 184. & $\begin{array}{l}\text { Institute of Diplomacy and Foreign } \\
\text { Relations (IDFR) }\end{array}$ & $\begin{array}{l}\text { Ministry of Foreign } \\
\text { Affairs }\end{array}$ \\
\hline 185. & $\begin{array}{l}\text { Southeast Asia Regional Centre for } \\
\text { Counter Terrorism (SEARCCT) }\end{array}$ & $\begin{array}{l}\text { Ministry of Foreign } \\
\text { Affairs }\end{array}$ \\
\hline 186. & $\begin{array}{l}\text { National Authority for Chemical } \\
\text { Weapons' Convention (NACWC) }\end{array}$ & $\begin{array}{l}\text { Ministry of Foreign } \\
\text { Affairs }\end{array}$ \\
\hline 187. & $\begin{array}{l}\text { Malaysia Healthcare Travel Council } \\
\text { (MHTC) }\end{array}$ & Ministry of Health \\
\hline 188. & Medical Device Authority (MDA) & Ministry of Health \\
\hline 189. & Institute for Medical Research (IMR) & Ministry of Health \\
\hline 190. & $\begin{array}{l}\text { Institute for Health Systems Research } \\
\text { (IHSR) }\end{array}$ & Ministry of Health \\
\hline 191. & Institute for Public Health (IKU) & Ministry of Health \\
\hline 192. & $\begin{array}{l}\text { Institute for Health Management } \\
\text { (IPK) }\end{array}$ & Ministry of Health \\
\hline 193. & $\begin{array}{l}\text { Institute for Respiratory Medicine } \\
\text { (IPR) }\end{array}$ & Ministry of Health \\
\hline 194. & Clinical Research Centre (CRC) & Ministry of Health \\
\hline 195. & $\begin{array}{l}\text { Institute for Health Behavioural } \\
\text { Research (IPTK) }\end{array}$ & Ministry of Health \\
\hline 196. & National Cancer Institute (NCl) & Ministry of Health \\
\hline
\end{tabular}




\begin{tabular}{|c|c|c|}
\hline 197. & National Blood Centre (PDN) & Ministry of Health \\
\hline 198. & $\begin{array}{l}\text { National Leprosy } \text { Control } \text { Centre } \\
\text { (PKKN) }\end{array}$ & Ministry of Health \\
\hline 199. & Children's Dental Centre & Ministry of Health \\
\hline 200. & $\begin{array}{l}\text { Eastern Sabah Security Command } \\
\text { (ESSCOM) }\end{array}$ & Ministry of Home Affairs \\
\hline 201. & Malaysian Civil Defense Force (APM) & Ministry of Home Affairs \\
\hline 202. & $\begin{array}{l}\text { Fire and Rescue Department of } \\
\text { Malaysia (JBPM) }\end{array}$ & Ministry of Home Affairs \\
\hline 203. & $\begin{array}{l}\text { Malaysian Immigration Department } \\
\text { (JIM) }\end{array}$ & Ministry of Home Affairs \\
\hline 204. & $\begin{array}{l}\text { Malaysian Maritime Enforcement } \\
\text { Agency (APMM) }\end{array}$ & Ministry of Home Affairs \\
\hline 205. & Malaysian Prison Department & Ministry of Home Affairs \\
\hline 206. & $\begin{array}{l}\text { Malaysian Volunteers' Department } \\
\text { (RELA) }\end{array}$ & Ministry of Home Affairs \\
\hline 207. & National Anti-Drug Agency (AADK) & Ministry of Home Affairs \\
\hline 208. & $\begin{array}{l}\text { National Registration Department } \\
\text { (JPN) }\end{array}$ & Ministry of Home Affairs \\
\hline 209. & Registry of Societies (ROS) & Ministry of Home Affairs \\
\hline 210. & Royal Malaysian Police (PDRM) & Ministry of Home Affairs \\
\hline 211. & $\begin{array}{l}\text { Percetakan Nasional Malaysia Berhad } \\
\text { (PNMB) }\end{array}$ & Ministry of Home Affairs \\
\hline 212. & $\begin{array}{l}\text { Film Censorship Board of Malaysia } \\
\text { (LPF) }\end{array}$ & Ministry of Home Affairs \\
\hline 213. & $\begin{array}{l}\text { Department of Local Governments } \\
\text { (JKT) }\end{array}$ & $\begin{array}{l}\text { Ministry of Housing and } \\
\text { Local Government }\end{array}$ \\
\hline 214. & Kampung Baru Division & $\begin{array}{l}\text { Ministry of Housing and } \\
\text { Local Government }\end{array}$ \\
\hline
\end{tabular}




\begin{tabular}{|c|c|c|}
\hline 215. & $\begin{array}{l}\text { National Landscape Department } \\
\text { (JLN) }\end{array}$ & $\begin{array}{l}\text { Ministry of Housing and } \\
\text { Local Government }\end{array}$ \\
\hline 216. & $\begin{array}{lll}\text { National Housing } & \text { Corporation } \\
\text { (PRIMA) } & & \\
\end{array}$ & $\begin{array}{l}\text { Ministry of Housing and } \\
\text { Local Government }\end{array}$ \\
\hline 217. & National Housing Department (JPN) & $\begin{array}{l}\text { Ministry of Housing and } \\
\text { Local Government }\end{array}$ \\
\hline 218. & $\begin{array}{l}\text { National Solid Waste Management } \\
\text { Department (JPSPN) }\end{array}$ & $\begin{array}{l}\text { Ministry of Housing and } \\
\text { Local Government }\end{array}$ \\
\hline 219. & $\begin{array}{l}\text { Urban and Rural Planning } \\
\text { Department of Peninsular } \\
\text { (JPBD) }\end{array}$ & $\begin{array}{l}\text { Ministry of Housing and } \\
\text { Local Government }\end{array}$ \\
\hline 220. & $\begin{array}{l}\text { Housing and Local Government } \\
\text { Training Institute (i-KPKT) }\end{array}$ & $\begin{array}{l}\text { Ministry of Housing and } \\
\text { Local Government }\end{array}$ \\
\hline 221. & $\begin{array}{l}\text { Solid Waste Management and Public } \\
\text { Cleansing Corporation (SWCorp) }\end{array}$ & $\begin{array}{l}\text { Ministry of Housing and } \\
\text { Local Government }\end{array}$ \\
\hline 222. & Perumahan Negara Bhd (SPNB) & $\begin{array}{l}\text { Ministry of Housing and } \\
\text { Local Government }\end{array}$ \\
\hline 223. & $\begin{array}{l}\text { Tribunal for Housing and Strata } \\
\text { Management (TPPS) }\end{array}$ & $\begin{array}{l}\text { Ministry of Housing and } \\
\text { Local Government }\end{array}$ \\
\hline 224. & $\begin{array}{l}\text { Department of Industrial Relations } \\
\text { (JPP) }\end{array}$ & $\begin{array}{l}\text { Ministry of Human } \\
\text { Resources }\end{array}$ \\
\hline 225. & Department of Manpower (JTM) & $\begin{array}{l}\text { Ministry of } \\
\text { Resources }\end{array}$ \\
\hline 226. & $\begin{array}{l}\text { Department of Occupational Safety } \\
\text { and Health (JKKP/DOSH) }\end{array}$ & $\begin{array}{l}\text { Ministry of } \\
\text { Resources }\end{array}$ \\
\hline 227. & $\begin{array}{l}\text { Department of Skills Development } \\
\text { (JPK/DSD) }\end{array}$ & $\begin{array}{l}\text { Ministry of } \\
\text { Resources }\end{array}$ \\
\hline 228. & $\begin{array}{l}\text { Department of Trade Union Affairs } \\
\text { (JHEKS) }\end{array}$ & $\begin{array}{l}\text { Ministry of Human } \\
\text { Resources }\end{array}$ \\
\hline
\end{tabular}


INTERNATIONAL JOURNAL OF ACADEMIC RESEARCH IN BUSINESS AND SOCIAL SCIENCES Vol. 9, No. 7, July, 2019, E-ISSN: 2222-6990 @ 2019 HRMARS

\begin{tabular}{|c|l|l|}
\hline 229. & Industrial Courts (MP) & $\begin{array}{l}\text { Ministry of Human } \\
\text { Resources }\end{array}$ \\
\hline 230. & $\begin{array}{l}\text { Sabah Labour Department (JTK } \\
\text { Sabah) }\end{array}$ & $\begin{array}{l}\text { Ministry of Human } \\
\text { Resources }\end{array}$ \\
\hline 231. & $\begin{array}{l}\text { Sarawak Labour Department (JTK } \\
\text { Sarawak) }\end{array}$ & $\begin{array}{l}\text { Ministry of Human } \\
\text { Resources }\end{array}$ \\
\hline 232. & $\begin{array}{l}\text { Human Resources Development } \\
\text { Fund** (HRDF/PSMB) }\end{array}$ & $\begin{array}{l}\text { Ministry of Human } \\
\text { Resources }\end{array}$ \\
\hline 233. & $\begin{array}{l}\text { National Institute of Occupational } \\
\text { Safety and Health (NIOSH) }\end{array}$ & $\begin{array}{l}\text { Ministry of Human } \\
\text { Resources }\end{array}$ \\
\hline 234. & $\begin{array}{l}\text { Skill Development Fund Corporation } \\
\text { (PTPK) }\end{array}$ & $\begin{array}{l}\text { Ministry of Human } \\
\text { Resources }\end{array}$ \\
\hline 235. & $\begin{array}{l}\text { Social Security Organisation } \\
\text { (PERKESO/SOCSO) }\end{array}$ & $\begin{array}{l}\text { Ministry of Human } \\
\text { Resources }\end{array}$ \\
\hline 236. & $\begin{array}{l}\text { Talent Corporation Malaysia Berhad } \\
\text { (TalentCorp) }\end{array}$ & $\begin{array}{l}\text { Ministry of Human } \\
\text { Resources }\end{array}$ \\
\hline 237. & $\begin{array}{l}\text { Malaysian Automotive Institute } \\
\text { (MAI) }\end{array}$ & $\begin{array}{l}\text { Ministry of International } \\
\text { Trade and Industry }\end{array}$ \\
\hline 238. & $\begin{array}{l}\text { Malaysian External } \\
\text { Development Trade } \\
\text { (MATRADE) }\end{array}$ & $\begin{array}{l}\text { Ministry of International } \\
\text { Trade and Industry }\end{array}$ \\
\hline 239. & $\begin{array}{l}\text { Malaysian Industrial Development } \\
\text { Finance (MIDF) }\end{array}$ & $\begin{array}{l}\text { Ministry of International } \\
\text { Trade and Industry }\end{array}$ \\
\hline 240. & $\begin{array}{l}\text { Malaysian Investment Development } \\
\text { Authority (MIDA) }\end{array}$ & $\begin{array}{l}\text { Ministry of International } \\
\text { Trade and Industry }\end{array}$ \\
\hline 241. & $\begin{array}{l}\text { Malaysian Productivity Corporation } \\
\text { (MPC) }\end{array}$ & $\begin{array}{l}\text { Ministry of International } \\
\text { Trade and Industry }\end{array}$ \\
\hline 242. & $\begin{array}{l}\text { Malaysian Steel Institute (MSI) } \\
\text { Ministry of International } \\
\text { rrade and Industry }\end{array}$ \\
\hline
\end{tabular}


INTERNATIONAL JOURNAL OF ACADEMIC RESEARCH IN BUSINESS AND SOCIAL SCIENCES Vol. 9, No. 7, July, 2019, E-ISSN: 2222-6990 @ 2019 HRMARS

\begin{tabular}{|c|c|c|}
\hline 243. & Malaysian Cocoa Board (LKM) & $\begin{array}{l}\text { Ministry of Primary } \\
\text { Industries }\end{array}$ \\
\hline 244. & Malaysian Palm Oil Board (MPOB) & $\begin{array}{l}\text { Ministry of } \\
\text { Industries }\end{array}$ \\
\hline 245. & Malaysian Pepper Board (MPB) & $\begin{array}{l}\text { Ministry of } \\
\text { Industries }\end{array}$ \\
\hline 246. & Malaysian Rubber Board (LGM) & $\begin{array}{l}\text { Ministry of Primary } \\
\text { Industries }\end{array}$ \\
\hline 247. & $\begin{array}{l}\text { Malaysian Timber Industries Board* } \\
\text { (MTIB) }\end{array}$ & $\begin{array}{l}\text { Ministry of } \\
\text { Industries }\end{array}$ \\
\hline 248. & $\begin{array}{l}\text { National Kenaf and Tobacco Board* } \\
\text { (LKTN) }\end{array}$ & $\begin{array}{l}\text { Ministry } \\
\text { Industries }\end{array}$ \\
\hline 249. & $\begin{array}{l}\text { Malaysian Furniture Promotion } \\
\text { Council (MFPC) }\end{array}$ & $\begin{array}{l}\text { Ministry of } \\
\text { Industries }\end{array}$ \\
\hline 250. & $\begin{array}{l}\text { Malaysian Palm Oil Certification } \\
\text { Council (MPOCC) }\end{array}$ & $\begin{array}{l}\text { Ministry of } \\
\text { Industries }\end{array}$ \\
\hline 251. & Malaysian Palm Oil Council (MPOC) & $\begin{array}{l}\text { Ministry of } \\
\text { Industries }\end{array}$ \\
\hline 252. & $\begin{array}{l}\text { Malaysian Rubber Export Promotion } \\
\text { Council (MREPC) }\end{array}$ & $\begin{array}{l}\text { Ministry of } \\
\text { Industries }\end{array}$ \\
\hline 253. & $\begin{array}{l}\text { Malaysian Timber Certification } \\
\text { Council (MTCC) }\end{array}$ & $\begin{array}{l}\text { Ministry of } \\
\text { Industries }\end{array}$ \\
\hline 254. & Malaysian Timber Council (MTC) & $\begin{array}{l}\text { Ministry of Primary } \\
\text { Industries }\end{array}$ \\
\hline 255. & $\begin{array}{ll}\text { Community } & \text { Development } \\
\text { Department (KEMAS) } & \\
\end{array}$ & $\begin{array}{l}\text { Ministry of } \\
\text { Development }\end{array}$ \\
\hline 256. & $\begin{array}{l}\text { Indigenous Community Affairs } \\
\text { Department (JAKOA) }\end{array}$ & $\begin{array}{l}\text { Ministry of } \\
\text { Development }\end{array}$ \\
\hline 257. & $\begin{array}{l}\text { Institute for Rural Advancement } \\
\text { (INFRA) }\end{array}$ & $\begin{array}{l}\text { Ministry of } \\
\text { Development }\end{array}$ \\
\hline
\end{tabular}


INTERNATIONAL JOURNAL OF ACADEMIC RESEARCH IN BUSINESS AND SOCIAL SCIENCES Vol. 9, No. 7, July, 2019, E-ISSN: 2222-6990 @ 2019 HRMARS

\begin{tabular}{|c|c|c|}
\hline 258. & $\begin{array}{l}\text { Central Terengganu Development } \\
\text { Authority (KETENGAH) }\end{array}$ & $\begin{array}{l}\text { Ministry of } \\
\text { Development }\end{array}$ \\
\hline 259. & $\begin{array}{l}\text { Kedah Regional Development } \\
\text { Authority (KEDA) }\end{array}$ & $\begin{array}{l}\text { Ministry of } \\
\text { Development }\end{array}$ \\
\hline 260. & $\begin{array}{l}\text { Rubber Industry Smallholders } \\
\text { Development Authority (RISDA) }\end{array}$ & $\begin{array}{l}\text { Ministry of } \\
\text { Development }\end{array}$ \\
\hline 261. & Majlis Amanah Rakyat (MARA) & $\begin{array}{l}\text { Ministry of } \\
\text { Development }\end{array}$ \\
\hline 262. & $\begin{array}{l}\text { Penang Regional } \\
\text { Authority (PERDA) }\end{array}$ & $\begin{array}{l}\text { Ministry of } \\
\text { Development }\end{array}$ \\
\hline 263. & $\begin{array}{lcr}\text { South Kelantan } & \text { Development } \\
\text { Authority (KESEDAR) } & \\
\end{array}$ & $\begin{array}{l}\text { Ministry of } \\
\text { Development }\end{array}$ \\
\hline 264. & $\begin{array}{l}\text { Southeast Johor Development } \\
\text { Authority (KEJORA) }\end{array}$ & $\begin{array}{l}\text { Ministry of } \\
\text { Development }\end{array}$ \\
\hline 265. & Islamic Tourism Centre (ITC) & $\begin{array}{l}\text { Ministry of Tourism, Art } \\
\text { and Culture }\end{array}$ \\
\hline 266. & Istana Budaya (IB) & $\begin{array}{l}\text { Ministry of Tourism, Art } \\
\text { and Culture }\end{array}$ \\
\hline 267. & $\begin{array}{l}\text { Malaysia Convention and Exhibition } \\
\text { Bureau (MyCEB) }\end{array}$ & $\begin{array}{l}\text { Ministry of Tourism, Art } \\
\text { and Culture }\end{array}$ \\
\hline 268. & $\begin{array}{l}\text { Malaysian Handicraft Development } \\
\text { Corporation (PKKM) }\end{array}$ & $\begin{array}{l}\text { Ministry of Tourism, Art } \\
\text { and Culture }\end{array}$ \\
\hline 269. & $\begin{array}{l}\text { National Culture and Art Department } \\
\text { (JKKN) }\end{array}$ & $\begin{array}{l}\text { Ministry of Tourism, Art } \\
\text { and Culture }\end{array}$ \\
\hline 270. & $\begin{array}{l}\text { National Culture, Art and Heritage } \\
\text { Academy (ASWARA) }\end{array}$ & $\begin{array}{l}\text { Ministry of Tourism, Art } \\
\text { and Culture }\end{array}$ \\
\hline 271. & National Heritage Department (JWN) & $\begin{array}{l}\text { Ministry of Tourism, Art } \\
\text { and Culture }\end{array}$ \\
\hline 272. & $\begin{array}{l}\text { National Visual Arts Development } \\
\text { Board (LPSVN) }\end{array}$ & $\begin{array}{l}\text { Ministry of Tourism, Art } \\
\text { and Culture }\end{array}$ \\
\hline
\end{tabular}


INTERNATIONAL JOURNAL OF ACADEMIC RESEARCH IN BUSINESS AND SOCIAL SCIENCES Vol. 9, No. 7, July, 2019, E-ISSN: 2222-6990 @ 2019 HRMARS

\begin{tabular}{|l|l|l|}
\hline 273. & Tourism Malaysia & $\begin{array}{l}\text { Ministry of Tourism, Art } \\
\text { and Culture }\end{array}$ \\
\hline 274. & $\begin{array}{l}\text { Department of Museums Malaysia } \\
\text { (JMM) }\end{array}$ & $\begin{array}{l}\text { Ministry of Tourism, Art } \\
\text { and Culture }\end{array}$ \\
\hline 275. & $\begin{array}{l}\text { National Archives of Malaysia (Arkib } \\
\text { Negara) }\end{array}$ & $\begin{array}{l}\text { Ministry of Tourism, Art } \\
\text { and Culture }\end{array}$ \\
\hline 276. & National Library of Malaysia (PNM) & $\begin{array}{l}\text { Ministry of Tourism, Art } \\
\text { and Culture }\end{array}$ \\
\hline 277. & Bintulu Port Authority (BPA) & Ministry of Transport \\
\hline 278. & $\begin{array}{l}\text { Civil Aviation Authority of Malaysia } \\
\text { (CAAM) }\end{array}$ & Ministry of Transport \\
\hline 279. & Johor Port Authority (LPJ) & Ministry of Transport \\
\hline 280. & Kuantan Port Authority (LPKTN) & Ministry of Transport \\
\hline 281. & Land Public Transport Agency (APAD) & Ministry of Transport \\
\hline 282. & $\begin{array}{l}\text { Malaysian Institute of Road Safety } \\
\text { Research (MIROS) }\end{array}$ & Ministry of Transport \\
\hline 283. & Marine Department of Malaysia \\
(JLM) & Ministry of Transport \\
\hline 284. & $\begin{array}{l}\text { Maritime Institute of Malaysia } \\
\text { (MIMA) }\end{array}$ & Ministry of Transport \\
\hline 285. & Penang Port Commission (SPPP/PPC) & Ministry of Transport \\
\hline 286. & Port Klang Authority (PKA) & Ministry of Transport \\
\hline 287. & $\begin{array}{l}\text { Railway Assets Corporation } \\
\text { (PAK/RAC) }\end{array}$ & Ministry of Transport \\
\hline 288. & Road Safety Department (JKJR) & Ministry of Transport \\
\hline 289. & Road Transport Department (JPJ) & Ministry of Transport \\
\hline 290. & $\begin{array}{l}\text { Sabah Commercial Vehicles Licensing } \\
\text { Board (LPKP Sabah) }\end{array}$ & Ministry of Transport \\
\hline 291. & $\begin{array}{l}\text { Sarawak Commercial Vehicles } \\
\text { Licensing Board (LPKP Sarawak) }\end{array}$ & Ministry of Transport \\
\hline
\end{tabular}




\begin{tabular}{|c|c|c|}
\hline 292. & $\begin{array}{l}\text { Department of Forestry of Peninsular } \\
\text { Malaysia (JPSM) }\end{array}$ & $\begin{array}{l}\text { Ministry of Water, Land } \\
\text { and Natural Resources }\end{array}$ \\
\hline 293. & $\begin{array}{l}\text { Department of Irrigation and } \\
\text { Drainage (JPS/DID) }\end{array}$ & $\begin{array}{l}\text { Ministry of Water, Land } \\
\text { and Natural Resources }\end{array}$ \\
\hline 294. & $\begin{array}{l}\text { Department of Marine Parks } \\
\text { (JTLM/DMPM) }\end{array}$ & $\begin{array}{l}\text { Ministry of Water, Land } \\
\text { and Natural Resources }\end{array}$ \\
\hline 295. & $\begin{array}{lr}\text { Department of Mineral and } \\
\text { Geoscience (JMG) }\end{array}$ & $\begin{array}{l}\text { Ministry of Water, Land } \\
\text { and Natural Resources }\end{array}$ \\
\hline 296. & $\begin{array}{l}\text { Department of the Director General } \\
\text { of Land and Mines (JKPTG) }\end{array}$ & $\begin{array}{l}\text { Ministry of Water, Land } \\
\text { and Natural Resources }\end{array}$ \\
\hline 297. & Department of Water Supply (JBA) & $\begin{array}{l}\text { Ministry of Water, Land } \\
\text { and Natural Resources }\end{array}$ \\
\hline 298. & $\begin{array}{l}\text { Department of Wildlife and National } \\
\text { Parks Peninsular Malaysia } \\
\text { (PERHILITAN) }\end{array}$ & $\begin{array}{l}\text { Ministry of Water, Land } \\
\text { and Natural Resources }\end{array}$ \\
\hline 299. & $\begin{array}{l}\text { Forestry Research Institute of } \\
\text { Malaysia (FRIM) }\end{array}$ & $\begin{array}{l}\text { Ministry of Water, Land } \\
\text { and Natural Resources }\end{array}$ \\
\hline 300. & $\begin{array}{l}\text { Malaysian Mapping and Survey } \\
\text { Department (JUPEM) }\end{array}$ & $\begin{array}{l}\text { Ministry of Water, Land } \\
\text { and Natural Resources }\end{array}$ \\
\hline 301. & $\begin{array}{l}\text { National Hydraulic Research Institute } \\
\text { of Malaysia (NAHRIM) }\end{array}$ & $\begin{array}{l}\text { Ministry of Water, Land } \\
\text { and Natural Resources }\end{array}$ \\
\hline 302. & $\begin{array}{l}\text { National Land and Survey Institute } \\
\text { (INSTUN) }\end{array}$ & $\begin{array}{l}\text { Ministry of Water, Land } \\
\text { and Natural Resources }\end{array}$ \\
\hline 303. & $\begin{array}{l}\text { National Water Services Commission } \\
\text { (SPAN) }\end{array}$ & $\begin{array}{l}\text { Ministry of Water, Land } \\
\text { and Natural Resources }\end{array}$ \\
\hline 304. & Sewerage Services Department (JPP) & $\begin{array}{l}\text { Ministry of Water, Land } \\
\text { and Natural Resources }\end{array}$ \\
\hline 305. & $\begin{array}{l}\text { National Family and Community } \\
\text { Development Board (LPPKN) }\end{array}$ & $\begin{array}{l}\text { Ministry of Women, } \\
\text { Family and Community } \\
\text { Development }\end{array}$ \\
\hline
\end{tabular}


INTERNATIONAL JOURNAL OF ACADEMIC RESEARCH IN BUSINESS AND SOCIAL SCIENCES

Vol. 9, No. 7, July, 2019, E-ISSN: 2222-6990 @ 2019 HRMARS

\begin{tabular}{|c|c|c|}
\hline 306. & $\begin{array}{l}\text { Community Welfare Department } \\
\text { (JKM) }\end{array}$ & $\begin{array}{l}\text { Ministry of Women, } \\
\text { Family and Community } \\
\text { Development }\end{array}$ \\
\hline 307. & $\begin{array}{l}\text { NAM Institute for the Empowerment } \\
\text { of Women (NIEW) }\end{array}$ & $\begin{array}{l}\text { Ministry of Women, } \\
\text { Family and Community } \\
\text { Development }\end{array}$ \\
\hline 308. & Institute of Social, Malaysia (ISM) & $\begin{array}{l}\text { Ministry of Women, } \\
\text { Family and Community } \\
\text { Development }\end{array}$ \\
\hline 309. & $\begin{array}{l}\text { Women Development Department } \\
\text { (JPW) }\end{array}$ & $\begin{array}{l}\text { Ministry of Women, } \\
\text { Family and Community } \\
\text { Development }\end{array}$ \\
\hline 310. & Public Works Department (JKR) & Ministry of Works \\
\hline 311. & $\begin{array}{l}\text { Construction Industry Development } \\
\text { Board (CIDB) }\end{array}$ & Ministry of Works \\
\hline 312. & Malaysian Highway Board (LLM) & Ministry of Works \\
\hline 313. & Board of Engineers of Malaysia (BEM) & Ministry of Works \\
\hline 314. & Board of Architects, Malaysia (LAM) & Ministry of Works \\
\hline 315. & Board of Surveyors of Malaysia (LTT) & Ministry of Works \\
\hline 316. & $\begin{array}{l}\text { Anti-Doping Agency of Malaysia } \\
\text { (ADAMAS) }\end{array}$ & $\begin{array}{l}\text { Ministry of Youth and } \\
\text { Sports }\end{array}$ \\
\hline 317. & Civic and Citizenship Bureau (BTN) & $\begin{array}{l}\text { Ministry of Youth and } \\
\text { Sports }\end{array}$ \\
\hline 318. & International Youth Centre (IYC) & $\begin{array}{l}\text { Ministry of Youth and } \\
\text { Sports }\end{array}$ \\
\hline 319. & $\begin{array}{l}\text { Malaysian Stadium Corporation } \\
\text { (PSM) }\end{array}$ & $\begin{array}{l}\text { Ministry of Youth and } \\
\text { Sports }\end{array}$ \\
\hline 320. & $\begin{array}{l}\text { Malaysian Youth Development } \\
\text { Research Institute (IPPBM) }\end{array}$ & $\begin{array}{l}\text { Ministry of Youth and } \\
\text { Sports }\end{array}$ \\
\hline
\end{tabular}


INTERNATIONAL JOURNAL OF ACADEMIC RESEARCH IN BUSINESS AND SOCIAL SCIENCES

Vol. 9, No. 7, July, 2019, E-ISSN: 2222-6990 @ 2019 HRMARS

\begin{tabular}{|l|l|l|}
\hline 321. & $\begin{array}{l}\text { National Service Training Department } \\
\text { (JLKN) }\end{array}$ & $\begin{array}{l}\text { Ministry of Youth and } \\
\text { Sports }\end{array}$ \\
\hline 322. & National Sports Council (MSN/NSC) & $\begin{array}{l}\text { Ministry of Youth and } \\
\text { Sports }\end{array}$ \\
\hline 323. & National Sports Institute (ISN) & $\begin{array}{l}\text { Ministry of Youth and } \\
\text { Sports }\end{array}$ \\
\hline 324. & $\begin{array}{l}\text { National Youth and Sports } \\
\text { Department (JBSN) }\end{array}$ & $\begin{array}{l}\text { Ministry of Youth and } \\
\text { Sports }\end{array}$ \\
\hline 325. & $\begin{array}{l}\text { Office of the Sports Commissioner } \\
\text { (PPS) }\end{array}$ & $\begin{array}{l}\text { Ministry of Youth and } \\
\text { Sports }\end{array}$ \\
\hline 326. & Registry of Youth Societies (ROY) & $\begin{array}{l}\text { Ministry of Youth and } \\
\text { Sports }\end{array}$ \\
\hline 327. & $\begin{array}{l}\text { Sepang International Circuit Sdn. } \\
\text { Bhd. (SIC) }\end{array}$ & $\begin{array}{l}\text { Ministry of Youth and } \\
\text { Sports }\end{array}$ \\
\hline 328. & $\begin{array}{l}\text { Subang Golf Course Corporation } \\
\text { (SGCC) }\end{array}$ & $\begin{array}{l}\text { Ministry of Youth and } \\
\text { Sports }\end{array}$ \\
\hline 329. & Education Service Commission (SPP) & Non-ministerial bodies \\
\hline 330. & Election Commission (SPR) (Website) & Non-ministerial bodies \\
\hline 331. & $\begin{array}{l}\text { Human Rights Commission } \\
\text { (SUHAKAM) }\end{array}$ & Non-ministerial bodies \\
\hline 332. & $\begin{array}{l}\text { Judicial Appointment Commission } \\
\text { (JAC) }\end{array}$ & Non-ministerial bodies \\
\hline 333. & $\begin{array}{l}\text { Malaysian Anti-Corruption } \\
\text { Commission (SPRM) }\end{array}$ & Non-ministerial bodies \\
\hline 334. & National Audit Department (JANM) & Non-ministerial bodies \\
\hline 335. & Public Service Commission (SPA) & Non-ministerial bodies \\
\hline 336. & Parliament & Non-ministerial bodies \\
\hline
\end{tabular}

SME Corp was established on 2 May 1996, is a Central Coordinating Agency operating its activities under the Ministry of International Trade and Industry Malaysia which plans the whole policies and action plans directed towards SME (SMEs) and coordination of the execution of developmental 
programs for SMEs through the several connected ministries and agencies. SME Corp. Malaysia is the agency driving the implementation of the SME Masterplan (2012-2020) that charts the policy direction of SME development until the year 2020. The Masterplan aims to accelerate the growth of SMEs via innovation-driven and productivity-led strategies for Malaysia to achieve a high-income nation status by 2020.

SME Corp. Malaysia is the nation's premier organization for the development of progressive SMEs to enhance wealth creation and social well-being of the nation. In fulfilling its mission of promoting the development of innovative, resilient and globally competitive SMEs through effective coordination and provision of business support, SME Corp. Malaysia endeavours to empower the critical mass of SMEs with efficient processes, robust business models, access to financial resources, smart partnerships, market-entry strategies and sustainable growth solutions, through various platforms and programs for SME development. Hence, by identifying the most related agencies which support entrepreneurs is very important to sustain and increasing Malaysia Gross Domestic Profits (GDP) before 2020. This study identified all related agencies in Malaysia Government as shown in table 2.

Table 2: The Government Agencies and Their Functions in Bumiputera Entrepreneurship Development

\begin{tabular}{|c|c|c|c|c|}
\hline No & AGENCIES & FUNCTIONS & SECTOR \& MINISTRY & SERVICES \\
\hline 2. & $\begin{array}{l}\text { Malaysian External } \\
\text { Trade Development } \\
\text { Corporation } \\
\text { (MATRADE) }\end{array}$ & $\begin{array}{l}\text { Assist local companies in promoting } \\
\text { their exports in order for them to } \\
\text { extend their reach to global markets. }\end{array}$ & $\begin{array}{ll}\text { Ministry } & \text { of } \\
\text { International Trade } \quad \& \\
\text { Industry (MITI) }\end{array}$ & $\begin{array}{l}\text { Generate a holistic database of facts on the } \\
\text { enhancement and development of trade; } \\
\text { Arranging training programs. }\end{array}$ \\
\hline 3. & $\begin{array}{l}\text { Malaysian Industrial } \\
\text { Development } \\
\text { Finance Berhad } \\
\text { (MIDF). }\end{array}$ & $\begin{array}{l}\text { Financing growth towards } \\
\text { manufacturing and services industry. }\end{array}$ & $\begin{array}{ll}\text { Ministry of } \\
\text { International Trade } \quad \& \\
\text { Industry (MITI) }\end{array}$ & $\begin{array}{l}\text { Hire purchase financing assistance; expansion } \\
\text { and diversification into several automotive } \\
\text { associated businesses. Focused on } \\
\text { manufacturing, automotive and services. }\end{array}$ \\
\hline 4. & $\begin{array}{l}\text { Malaysia } \\
\text { Productivity } \\
\text { Corporation (MPC) }\end{array}$ & $\begin{array}{l}\text { Leading in the distribution of } \\
\text { information associated with } \\
\text { productivity and issues; serves as data }\end{array}$ & $\begin{array}{ll}\text { Ministry of } \\
\text { International Trade } \quad \text { \& } \\
\text { Industry (MITI) }\end{array}$ & $\begin{array}{l}\text { To produce own experienced personnel in } \\
\text { productivity, quality, management and } \\
\text { entrepreneurship; giving guidance and } \\
\text { coordinating the executuion of programmes and }\end{array}$ \\
\hline
\end{tabular}




\begin{tabular}{|c|c|c|c|c|}
\hline & & $\begin{array}{l}\text { and reference point for productivity } \\
\text { indices related to Malaysia. }\end{array}$ & & $\begin{array}{l}\text { activities to do with productivity and quality; } \\
\text { evaluating and certification of training programs } \\
\text { supervision and management, entrepreneurship } \\
\text { development programs and productivity and } \\
\text { quality management programs done by the } \\
\text { private agencies to the public; carrying out } \\
\text { training or related programmes dealing on } \\
\text { productivity, quality, management and } \\
\text { entrepreneurship; offering consultancy services } \\
\text { on aspects of to productivity, quality, } \\
\text { management and entrepreneurship. }\end{array}$ \\
\hline 5. & SME BANK. & $\begin{array}{l}\text { Provision of financing, treasury \& } \\
\text { investment, and Entrepreneur } \\
\text { development. }\end{array}$ & $\begin{array}{l}\text { Ministry of Domestic } \\
\text { trade, Cooperatives and } \\
\text { Consumerism (KPDNKK) }\end{array}$ & $\begin{array}{l}\text { SME Bank Online Directory; provision of factory } \\
\text { lots for rent, financial support, entrepreneurial } \\
\text { training, advisory services and/or technical } \\
\text { support and operational efficiency. }\end{array}$ \\
\hline 6. & $\begin{array}{l}\text { Halal Industry } \\
\text { Development } \\
\text { Corporation (HDC) }\end{array}$ & $\begin{array}{l}\text { To spearhead the development of } \\
\text { Halal benchmark, audit and } \\
\text { certification process to guard the } \\
\text { sanctity of Halal; To facilitate the flow } \\
\text { of investment into the Halal industry in } \\
\text { Malaysia; To promote the growth and } \\
\text { involvement of Malaysian firms in the } \\
\text { world Halal market; and To develop, } \\
\text { foster, and promote the Malaysian } \\
\text { Halal brand. }\end{array}$ & $\begin{array}{ll}\text { Ministry of } \\
\text { International Trade } \quad \& \\
\text { Industry (MITI) }\end{array}$ & $\begin{array}{l}\text { Oversee the general activities involved in } \\
\text { developing the Halal industry, which focuses on } \\
\text { maintaining Halal benchmarks, certification, and } \\
\text { capacity building for the Halal merchandise. The } \\
\text { corporation encourages involvement of local } \\
\text { businesses in the world Halal market and } \\
\text { nurture the growth of Malaysian firms globally. } \\
\text { The corporation gives manufacturers, channels, } \\
\text { entrepreneurs, academicians, and business } \\
\text { investors, the required support to enter the } \\
\text { world Halal market by using the Malaysian } \\
\text { standard and practice in food and non-food } \\
\text { sector. }\end{array}$ \\
\hline 7. & $\begin{array}{l}\text { Malaysian } \\
\text { Investment }\end{array}$ & $\begin{array}{l}\text { Supports firms that are willing to } \\
\text { venture into the manufacturing and } \\
\text { services sectors. }\end{array}$ & $\begin{array}{ll}\text { Ministry } & \text { of } \\
\text { International Trade } & \& \\
\text { Industry (MITI) } & \end{array}$ & $\begin{array}{l}\text { Offering information on the opportunities open } \\
\text { to investors, and help in building joint venture }\end{array}$ \\
\hline
\end{tabular}




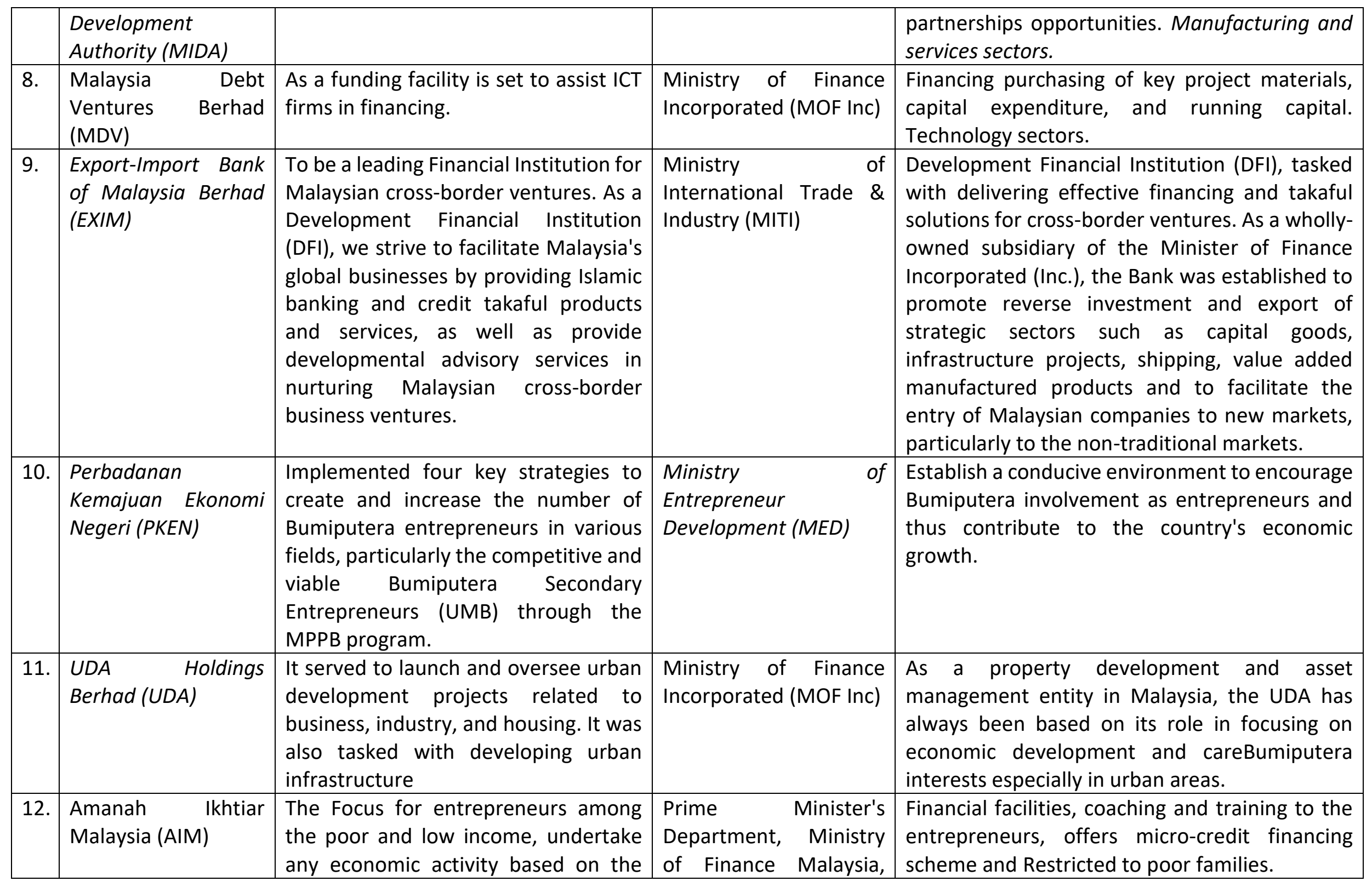


INTERNATIONAL JOURNAL OF ACADEMIC RESEARCH IN BUSINESS AND SOCIAL SCIENCES Vol. 9, No. 7, July, 2019, E-ISSN: 2222-6990 @ 2019 HRMARS

\begin{tabular}{|c|c|c|c|c|}
\hline & & $\begin{array}{l}\text { skills acquired as an effort to increase } \\
\text { family income. }\end{array}$ & $\begin{array}{l}\text { Ministry of Rural and } \\
\text { Regional Development, } \\
\text { representatives of the } \\
\text { Selangor State } \\
\text { Government }\end{array}$ & \\
\hline 13. & $\begin{array}{l}\text { Yayasan } \\
\text { Pembangunan } \\
\text { Ekonomi Islam } \\
\text { Malaysia (YaPEIM) }\end{array}$ & $\begin{array}{l}\text { Social Business \& Business - } \\
\text { Implementation of competitive, } \\
\text { innovative, efficient, efficient and } \\
\text { quality Shariah compliant business } \\
\text { social programs that impact on } \\
\text { socioeconomic development \& social } \\
\text { well-being through the provision of } \\
\text { micro credit financing facilities, } \\
\text { platforms and support for } \\
\text { entrepreneurial development. } \\
\text { Implementing profitable business } \\
\text { activities for the institution to } \\
\text { strengthen the institutional position } \\
\text { through efficient } \\
\text { Management and Administration and } \\
\text { to practice good Governance } \\
\text { practices; and Society \& Government } \\
\text { Development - Cultivate and provide } \\
\text { easy and reliable infrastructure for the } \\
\text { practicing community. Providing } \\
\text { charitable donations to the beneficiary } \\
\text { in a variety of transparent and } \\
\text { effective and beneficial ways to the } \\
\text { community. }\end{array}$ & $\begin{array}{ll}\text { Prime } & \text { Minister's } \\
\text { Department } & \end{array}$ & $\begin{array}{l}\text { To mobilize its potential and expand its role, } \\
\text { YaPEIM was established and relaunched on July } \\
\text { 20, } 1984 \text { by Y.A.B. Prime Minister at that time. } \\
\text { YaPEIM is administered by the Board of Trustees } \\
\text { through the YaPEIM Trust Deed. The leading } \\
\text { institutions lead socioeconomic development } \\
\text { inclusive and effective for the well-being of the } \\
\text { people. }\end{array}$ \\
\hline
\end{tabular}




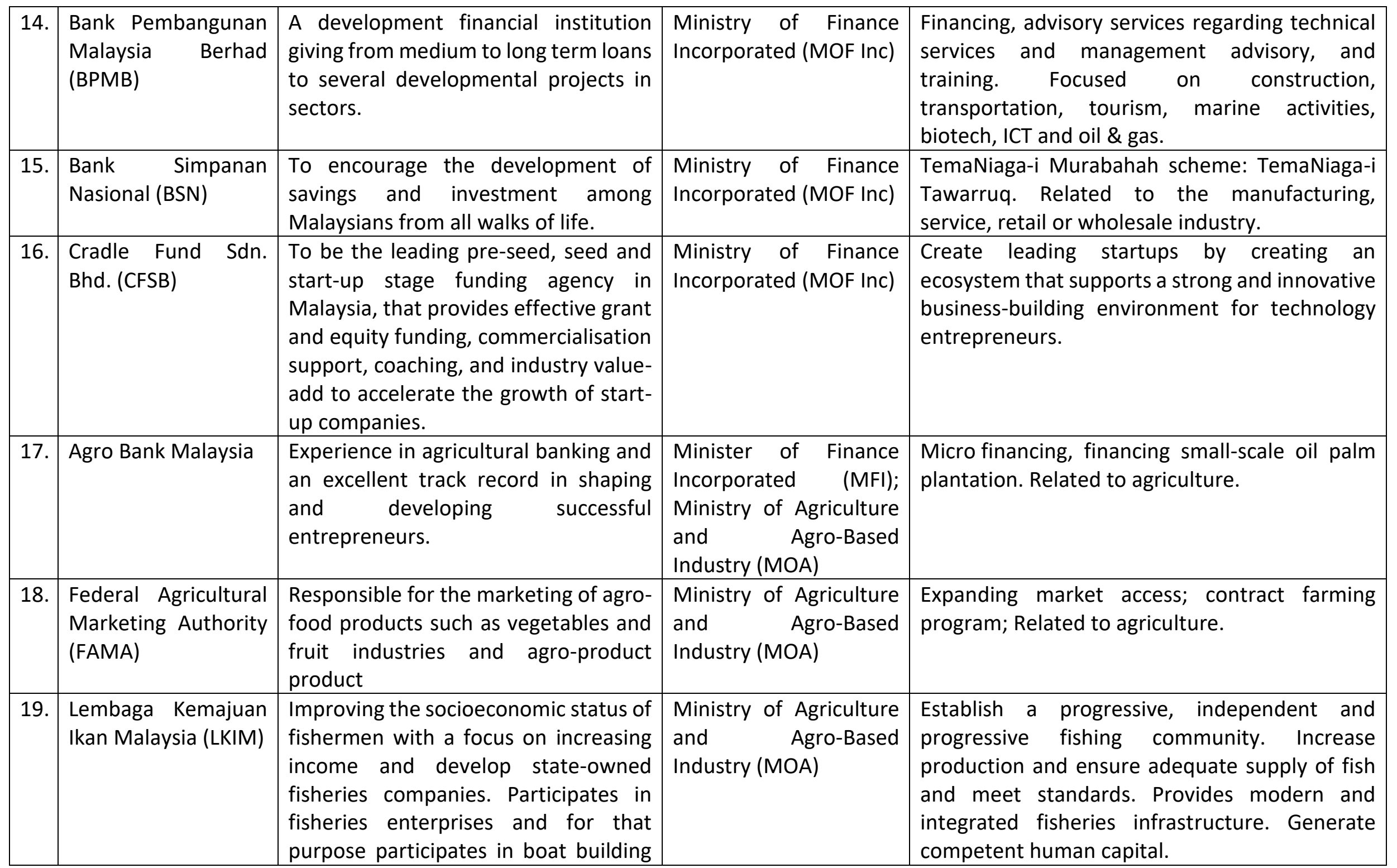




\begin{tabular}{|c|c|c|c|c|}
\hline & & $\begin{array}{l}\text { and production of fish supplies and } \\
\text { equipment. }\end{array}$ & & \\
\hline 21. & $\begin{array}{l}\text { Malaysian } \\
\text { Agricultural } \\
\text { Research and } \\
\text { Development } \\
\text { Institute (MARDI) } \\
\end{array}$ & $\begin{array}{l}\text { Develop the indigenous science and } \\
\text { technology capabilities in support of } \\
\text { the development and modernization } \\
\text { of the national food and agriculture } \\
\text { sector. }\end{array}$ & $\begin{array}{l}\text { Ministry of Agriculture } \\
\text { and } \quad \text { Agro-Based } \\
\text { Industry (MOA) }\end{array}$ & $\begin{array}{l}\text { Training, and providing grant aids for research in } \\
\text { agriculture. Research activities related to } \\
\text { agriculture. }\end{array}$ \\
\hline 23. & $\begin{array}{l}\text { Majlis Rakyat } \\
\text { Amanah (MARA). }\end{array}$ & $\begin{array}{l}\text { Supporting, training, and directing the } \\
\text { business and industrial affairs of of } \\
\text { Bumiputera. }\end{array}$ & $\begin{array}{ll}\text { Ministry } & \text { of } \\
\text { Entrepreneur } & \\
\text { Development (MED) }\end{array}$ & $\begin{array}{l}\text { Business Financing; Entrepreneur, Training. } \\
\text { Business Consultation, Service. } \\
\text { Marketing Development; TechnoEntrepreneur } \\
\text { Development; } \\
\text { Entrepreneur Development Division; Industrial } \\
\text { and Infrastructure Development, Education. }\end{array}$ \\
\hline
\end{tabular}




\begin{tabular}{|c|c|c|c|c|}
\hline 24. & $\begin{array}{l}\text { Perbadanan } \\
\text { Nasional Usahawan } \\
\text { Berhad (PUNB) }\end{array}$ & $\begin{array}{l}\text { Develop Bumiputera entrepreneurs in } \\
\text { wholesale sectors that provide bulk } \\
\text { purchase activities or big-scale } \\
\text { retaining; standardising brands, } \\
\text { product arrangements. }\end{array}$ & $\begin{array}{l}\text { Ministry of Finance } \\
\text { Incorporated (MOF Inc) }\end{array}$ & $\begin{array}{l}\text { Consulting \& Monitoring Services; the financing } \\
\text { provision, PUNB similarly offers trainings; } \\
\text { marketing and promotion; corporate } \\
\text { governance; Business Development. }\end{array}$ \\
\hline 25. & $\begin{array}{l}\text { National SMEs } \\
\text { Development } \\
\text { Council (NSDC) }\end{array}$ & $\begin{array}{l}\text { Decision making affairs and activities } \\
\text { regarding the development of SMEs in } \\
\text { Malaysia and to ensure all round as } \\
\text { well as coordinated developmental } \\
\text { activities of SMEs through several } \\
\text { sectors. }\end{array}$ & $\begin{array}{lr}\text { Ministry of } & \text { Finance as } \\
\text { Public } & \text { Private } \\
\text { Partnership } & \text { (PPP) } \\
\text { model } & \end{array}$ & $\begin{array}{l}\text { Planning, revising, and stressing the agenda of } \\
\text { Bumiputera development. }\end{array}$ \\
\hline 26. & $\begin{array}{l}\text { Tabung Economi } \\
\text { Kumpulan Usahawan } \\
\text { Niaga (TEKUN). }\end{array}$ & $\begin{array}{l}\text { Provide financing facilities to } \\
\text { Bumiputera easy and quick to start and } \\
\text { develop their businesses. }\end{array}$ & $\begin{array}{l}\text { Ministry of } \text { Agriculture } \\
\text { and } \quad \text { Agro-Based } \\
\text { Industry (MOA) }\end{array}$ & $\begin{array}{l}\text { Business development and not only provide } \\
\text { business capital alone; business opportunities; } \\
\text { capital financing business, guidance and support } \\
\text { and Entrepreneurs Network. }\end{array}$ \\
\hline 27. & $\begin{array}{l}\text { Small and Medium } \\
\text { Industries } \\
\text { Development } \\
\text { Corporation } \\
\text { (SMIDEC) } \\
\end{array}$ & $\begin{array}{l}\text { Further promote the development of } \\
\text { Small and Medium Industries (SMIs) or } \\
\text { enterprises (SMEs) in the } \\
\text { manufacturing sector. }\end{array}$ & $\begin{array}{l}\text { Ministry of } \\
\text { International Trade and } \\
\text { Industry (MITI) }\end{array}$ & $\begin{array}{l}\text { Advisory services, fiscal and financial assistance, } \\
\text { infrastructural facilities, market access and other } \\
\text { support programs. Specific to manufacturing. }\end{array}$ \\
\hline 28. & $\begin{array}{|ll|}\text { Perbadanan } & \\
\text { Nasional } & \text { Berhad } \\
\text { (PNS) } & \\
\end{array}$ & $\begin{array}{l}\text { Lead the development of Malaysia's } \\
\text { franchise industry. }\end{array}$ & $\begin{array}{ll}\text { Ministry of } \\
\text { Entrepreneur } \\
\text { Development (MED) }\end{array}$ & $\begin{array}{l}\text { Franchising development. Specific to } \\
\text { franchising. }\end{array}$ \\
\hline 29. & \begin{tabular}{|lr} 
Permodalan & \\
Nasional & Berhad \\
(National & Equity \\
Corporation) & (PNB) \\
\end{tabular} & $\begin{array}{l}\text { To promote shared ownership in the } \\
\text { corporate sector. }\end{array}$ & $\begin{array}{l}\text { Corporate sector. } \\
\text { Government-linked } \\
\text { investment company }\end{array}$ & $\begin{array}{l}\text { Asset management, Unit Trust \& REIT, Property } \\
\text { \& Hospitality, Education \& CSR. Focused on asset } \\
\text { management. }\end{array}$ \\
\hline 30. & $\begin{array}{l}\text { Malaysian Industrial } \\
\text { Development } \\
\text { Finance Berhad } \\
\text { (MIDF) }\end{array}$ & $\begin{array}{l}\text { MIDF is aimed at expanding its } \\
\text { supports to SMEs by extending grants } \\
\text { or funding as low as } \$ 50,000 ; \text { hire } \\
\text { purchase financing assistance for }\end{array}$ & $\begin{array}{l}\text { Ministry of } \\
\text { International Trade and } \\
\text { Industry (MITI) }\end{array}$ & $\begin{array}{l}\text { Aimed at financing growth towards } \\
\text { manufacturing and services industry. }\end{array}$ \\
\hline
\end{tabular}




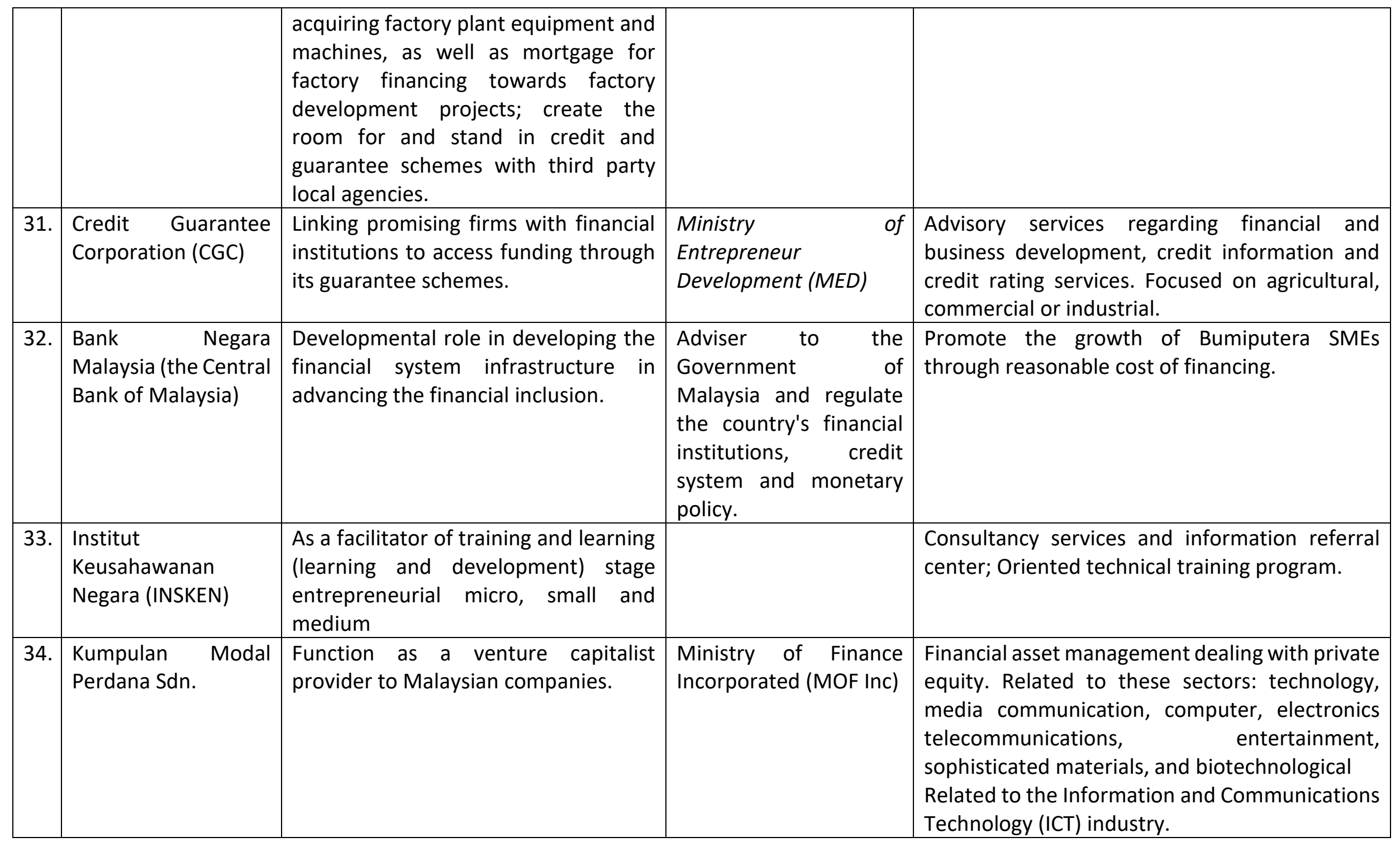




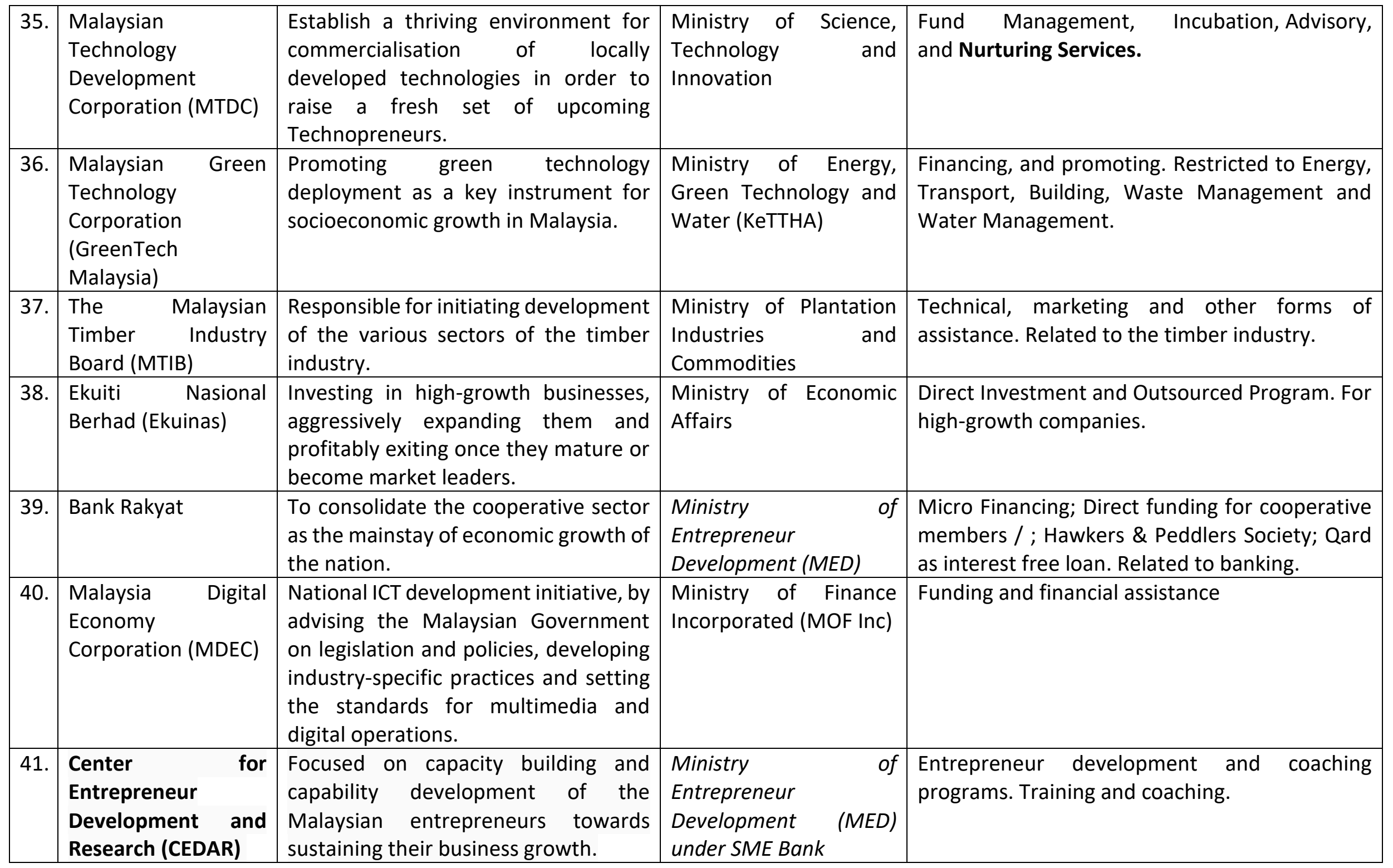




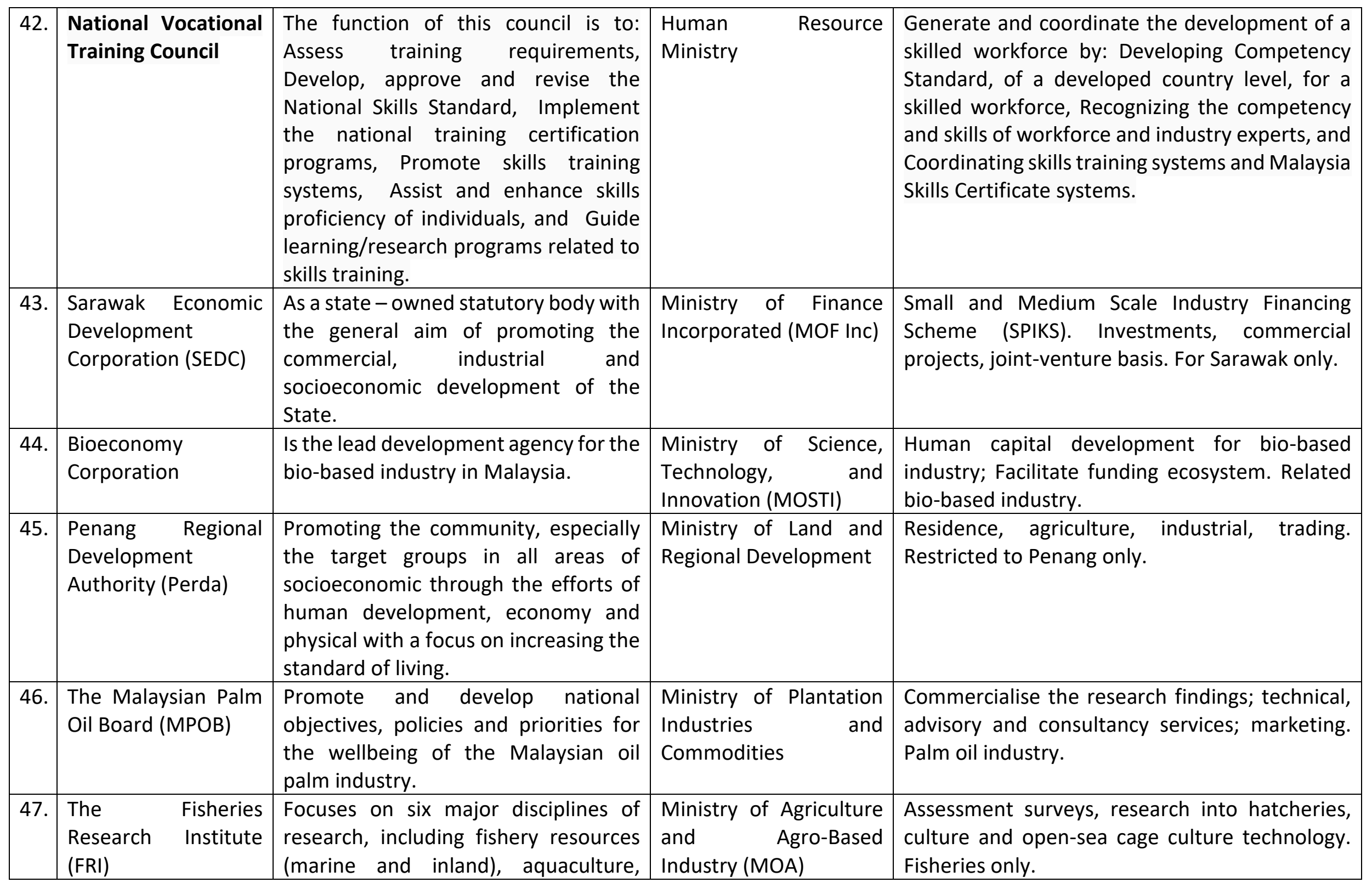




\begin{tabular}{|c|c|c|c|c|}
\hline & & $\begin{array}{l}\text { aquatic ecology, biotechnology, } \\
\text { fisheries product development and } \\
\text { fish health. }\end{array}$ & & \\
\hline 48. & MIMOS Berhad & $\begin{array}{l}\text { Forefront technology provider in } \\
\text { Information and Communications } \\
\text { Technology, Industrial Electronics } \\
\text { Technology and Nano-Semiconductor } \\
\text { Technology }\end{array}$ & $\begin{array}{l}\text { Ministry of Science, } \\
\text { Technology and } \\
\text { Innovation (MOSTI) }\end{array}$ & $\begin{array}{l}\text { Smart partnerships and inclusive growth models } \\
\text { and strategies; creation for Malaysian } \\
\text { technopreneurs through patentable technology } \\
\text { platforms. Related to technology based } \\
\text { industries. }\end{array}$ \\
\hline 50. & $\begin{array}{l}\text { Malaysia Automative } \\
\text { Institute (MAI) }\end{array}$ & $\begin{array}{l}\text { Focus extensively in acquiring and } \\
\text { transferring technologies associated } \\
\text { with automotive development. }\end{array}$ & $\begin{array}{l}\text { Ministry of } \\
\text { International Trade and } \\
\text { Industry (MITI) }\end{array}$ & $\begin{array}{l}\text { Advice and assistance in formulating strategies. } \\
\text { Related to automative. }\end{array}$ \\
\hline 51. & $\begin{array}{ll}\text { Technology } & \text { Park } \\
\text { Malaysia } & \end{array}$ & $\begin{array}{l}\text { Maximizing of its land assets, } \\
\text { enhancing its commercialization and } \\
\text { support services, increasing the } \\
\text { efficacy of its advanced infrastructure } \\
\text { and networking capabilities, } \\
\text { developing start-up friendly policies } \\
\text { for technology based quality start-up } \\
\text { companies with global potential. }\end{array}$ & $\begin{array}{l}\text { Ministry of Finance } \\
\text { Incorporated (MOF Inc) }\end{array}$ & $\begin{array}{l}\text { Rental of incubator premises; Technology and } \\
\text { business incubation programs; Technology } \\
\text { commercialisation assistance and support. } \\
\text { Related to Technology based. }\end{array}$ \\
\hline 52. & $\begin{array}{l}\text { Malaysian Steel } \\
\text { Institute (MISI) }\end{array}$ & 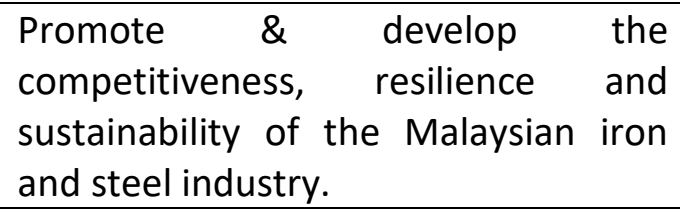 & $\begin{array}{l}\text { Ministry of } \\
\text { International Trade and } \\
\text { Industry (MITI) }\end{array}$ & $\begin{array}{l}\text { Advisory services; standards in consultation with } \\
\text { the relevant government agencies. Related to } \\
\text { iron and steel. }\end{array}$ \\
\hline 53. & $\begin{array}{l}\text { Construction } \\
\text { Industry } \\
\text { Development Board } \\
\text { (CIDB) }\end{array}$ & $\begin{array}{l}\text { To enhance the competitiveness of the } \\
\text { Malaysian construction industry. }\end{array}$ & $\begin{array}{l}\text { Ministry of Works and } \\
\text { Public Amenities }\end{array}$ & Restricted to construction. \\
\hline
\end{tabular}


INTERNATIONAL JOURNAL OF ACADEMIC RESEARCH IN BUSINESS AND SOCIAL SCIENCES Vol. 9, No. 7, July, 2019, E-ISSN: 2222-6990 @ 2019 HRMARS

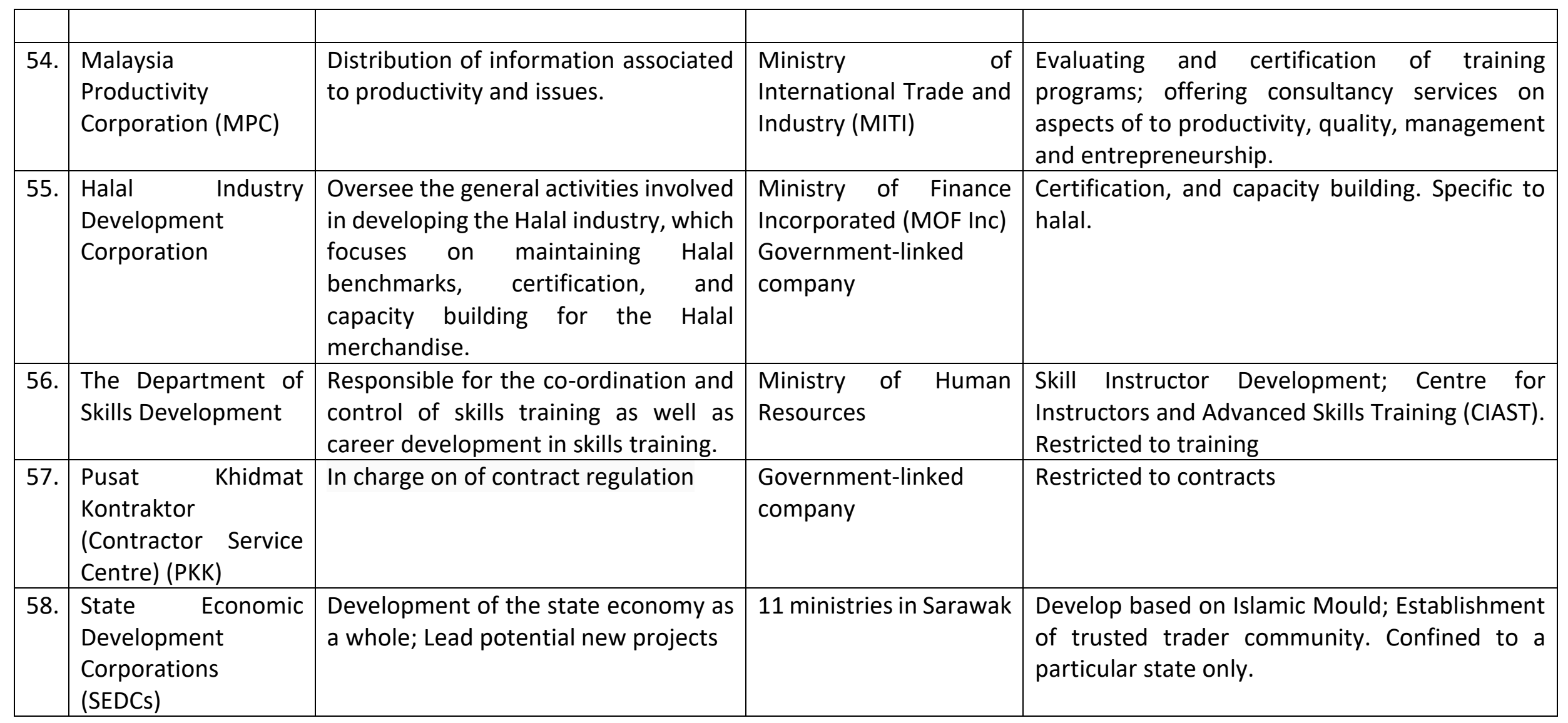

Forming an entrepreneurial society among the Bumiputera is a concerted effort of several agencies (Ministry, 2017). In 1990s, the Ministry of Trade and Industry formed the Bumiputera Participation Division that serves as business advisory board to guide some selected Bumiputera entrepreneurs recognised with potentials into the right venture that suits them. So also, these agencies were established to offer support services such as: the Export Trade Centre that exposes the entrepreneurs to foreign opportunities, the National Productivity Centre charged with providing trainings based on management, which are conducted in collaboration with the Malaysian Entrepreneur Development Centre and National Entrepreneurship Research Development Association. The Development Bank of Malaysia, the Agricultural Bank of Malaysia, the commercial banks through directive of the Central Bank of Malaysia were also involved in financing and lending schemes to the Bumiputera entrepreneurs (Ministry, 2017). 
Soon \& Huat, (1999) Forming an entrepreneurial society among the Bumiputera is a concerted effort of several agencies. In 1990s, the Ministry of Trade and Industry formed the Bumiputera Participation Division that serves as a business advisory board to guide some selected Bumiputera entrepreneurs recognized with potentials into the right venture that suits them. So also, these agencies were established to offer auxiliary services such as: the Export Trade Centre that exposes the entrepreneurs to foreign opportunities, the National Productivity Centre charged with providing trainings based on management, which are conducted in collaboration with the Malaysian Entrepreneur Development Centre and National Entrepreneurship Research Development Association. The Development Bank of Malaysia, the Agricultural Bank of Malaysia, the commercial banks through directive of the Central Bank of Malaysia were also involved in financing and lending schemes to the Bumiputera entrepreneurs (Soon \& Huat, 1999). Similarly, Perbadanan Usahawan Nasional Bhd assisted the Bumiputera entrepreneurs' attempt to start franchising business by allocating a sum of RM100 million. Equally, the New Entrepreneurs Fund was launched in December 1989 to stimulate new enterprises of Bumiputera that open up into manufacturing, agriculture, tourism and export-oriented industries. The implementation of various programs has been entrusted to several government agencies and lately, government departments, as well, including MARA (Council of Trust for the Indigenous People), UDA (Urban Development Authority), PERNAS (National Trading Corporation), and others, such as MIDF (Malaysian Industrial Development Finance), NPC (National Productivity Centre), FIDA (Federal Industrial Development Authority), CGC (Credit Guarantee Corporation), 
MIEL Malaysian Tnclustrial Estate (Amir, 1983). ASN (National Share Trust Board), Bank Pembangunan (Development Bank), and Bank Bumiputra (Bumiputra Bank). Similar roles are also given to the SEDCs (State Economic Development Corporat ions) set up in each of the thirteen states of Malaysia. The main agencies, however, are MARA, UDA, and PERNAS according to Hashim.

Zulkifli, Omar, Mohd, \& Azmi, (2015) These are agencies that facilitate Bumiputera entrepreneurs' creation and growth in Malaysia: Majlis Amanah Rakyat (MARA), Perbadanan Nasional Berhad (PNS), SME Bank, Tabung Ekonomi Usaha Niaga (TEKUN), Bank Rakyat, Bank Pembangunan and UDA Holdings Bhd. The People's Trustee Council (MARA) was set up in 1966 to encourage the small enterprises and support them financially and provide infrastructural developments to them. The agency helps pinpoint business or investments with potentials for Bumiputera, assists them with technical or commercial help, while constructing business units and lots for Bumiputera to occupy at affordable rates, as well as grow some businesses which can later be acquired by the Bumiputera entrepreneurs to expand their shareholding (Ministry, 2017). According to Zulkifli, Omar, Mohd, \& Azmi, (2015) Majlis Amanah Rakyat (MARA) is set to promote entrepreneurship training programs, market development program and so on. The administration and regulations imposed on accessing loans should be relaxed so that it would encourage more Bumiputera entrepreneurs to join the business environment and could as well deter Bumiputera entrepreneurs obtaining loans from unlicensed agents.

"A major obstacle faced by new Bumiputera entrepreneurs is that of obtaining adequate finance at reasonable cost. Hence, The Credit Guarantee Corp Malaysia Bhd (CGC) was established to serve as a link between the financial houses and Bumiputera entrepreneurs by providing a guaranteeing system of ensuring solvency to win the confidence of lenders by CGC in their ability to fulfil obligations and to provide lower interest rates on loans compared to commercial bank rates (Ariff \& Abubakar, 2007).

Ariff \& Abubakar, (2007) "Under the new scheme, Bumiputera entrepreneurs can obtain financing from participating financial institutions, with the assistance of the Credit Guarantee Corp Malaysia Bhd (CGC)," he told reporters in Kuala Lumpur yesterday after attending a dialogue forum organised by the Malaysian Women's Advancement Institute, Association of Bumiputera Women Entrepreneurs Malaysia and Wanita Umno.

Similarly, Perbadanan Usahawan Nasional Bhd assisted the Bumiputra entrepeneurs' attempt to star franchising business by allocating a sum of RM100 million. Equally, the New Entrepreneurs Fund was launched in December 1989 to stimulate new enterprises of Bumiputera that open up into manufacturing, agriculture, tourism and export-oriented industries. "The interest rate on such loans was set at only 5 per cent per year. Because of this, the rate of utilisation of the credit facility was very encouraging and a year or two later, the New Entrepreneurs Fund was fully utilised," he explained. The Credit Guarantee Corp Malaysia Bhd (CGC) was established to serve as a link between the financing houses and Bumiputera entrepreneurs by providing a guaranteeing system of ensuring solvency to win the confidence of lenders by CGC in their ability to fulfil obligations and to provide lower interest rates on loans compared to commercial banks rates (Ariff \& Abubakar, 2007).

Whah \& Guan, (2015) argue in this context, a new private equity fund management company called Ekuiti Nasional Berhad (Ekuinas) was established in September 2009 to realize the objectives of the NEM. It was given the mandate to create the next generation of leading Malaysian companies by undertaking private equity investments in high potential local businesses. Its investment targets include high potential Bumiputera and non-Bumiputera companies, and the acquisition of Malaysian 
companies, non-core GLC assets, public listed companies, multinational corporations and trust foundations (EKUINAS, 2014).

"The interest rate on such loans was set at only 5 per cent per year. Because of this, the rate of utilisation of the credit facility was very encouraging and a year or two later, the New Entrepreneurs Fund was fully utilised," he explained. Ahmad \& Hoffman, (2007) framework of assessing and measuring entrepreneurship recognized these three independent but inter-related flows, which are: "determinants", "entrepreneurial performance", and, "impact"; that are useful in the establishment, evaluation and assessment of policy measures.

According to Ghosh, Liang, Meng, \& Chan, (2001), KS Fs are defined as factors critical to excellent performance of the company rather than survival. Amir, (1983) described success as effectiveness of enterprises in accomplishing their objectives. The success factors that were identified are satisfying customer needs, close working relationship between top management and employee, regionalization, leadership, availability of financial and technology resources, and support (Hung \& Effendi, 2011). Entrepreneurial performance evaluates the entrepreneurial activities engaged by the entrepreneurs which are key in executing the impacts. These impacts are valuable outcomes generated from the actions of the entrepreneurs or brought by entrepreneurship which is reflected through significant economic growth, employment opportunities, or the income distribution of the people. As emphasized by Ahmad \& Hoffman, (2007) a country has the freewill to decide which kind of performance indicators to look into in line with their policy aims.

\section{Conclusion}

Therefore, to analyses the performance index of Bumiputera entrepreneurs may require that we reflect back to the erstwhile policies, especially the New Economic Policy (1970 - 1990) that centered around the objectives of restructuring the society in general by enhancing the economic activities of all races; poverty eradication especially for the Bumiputera; and importantly, developing the entrepreneurial capabilities of the Bumiputera in the commercial arena. Multitudes of inherent environmental and sociological elements as well as individual features of the entrepreneurs may bear upon the results gained from the entrepreneurial activity. The key social and economic impacts aimed through entrepreneurship considering the framework were noted as employment, economic growth and poverty reduction.

In fact, in relative terms, based on the size of their capital, the Bumiputera firms in Malaysia can be said to act as better sources of employment compared to their counterparts in developed countries who are endowed with larger capital, but who are by definition small businesses into business without any kind of business training and, of those who finally established themselves, more than half of them remained without training (Amir, 1983). The Bumiputera firms were very small business ventures by any standard at all, but relative to the size of their assets they employed more people than smaller firms in developed countries such as Japan, the U.S .A., Brita in, Australia or New Zealand. The Impact analysis Framework on SME Development Programs (IAFSP) has been adopted since early of this decade to evaluate the performance of SME developmental programs, which was certified by the NSCD in order to regularise the application of common KPIs (SME, 2016).

\section{Acknowledgement}

The authors would like to express an appreciation to the FRGS grant RR206, Universiti Sultan Zainal Abidin (UniSZA) for giving advice, guidance, and their research facilities. Authors also would like to acknowledge a great support from Siti Nur Khadijah binti Yahya for this project. 


\section{References}

Ahmad, N., \& Hoffman, A. (2007). A framework for addressing and measuring entrepreneurship. Oecd, 2(November), 1-4,6-21,23,25-29,31-36. https://doi.org/10.1787/243160627270

Alom, F., Abdullah, M. A., Moten, A. R., \& Azam, S. M. F. (2016). Success factors of overall improvement of microenterprises in Malaysia: an empirical study. Journal of Global Entrepreneurship Research, 6(1), 7. https://doi.org/10.1186/s40497016-0050-2

Amir, H. (1983). Bumiputera Entrepreneurship.

Anthony, S., \& Syahira, H. (2009). The Contradiction of Entrepreneurship through Affirmative Action: The Case of Malaysia. The Copenhagen Journal of Asian Studies, 27(1), 38-66. Retrieved from rauli.cbs.dk/index.php/cjas/article/view/2217

Ariff, M., \& Abubakar, S. Y. (2007). the Malaysian Financial Crisis: Economic Impact and Recovery Prospects. The Developing Economies, 37(4), 417-438. https://doi.org/10.1111/j.1746-1049.1999.tb00241.x

Ariff, M., \& Abubakar, S. Y. (2015). Corporate entrepreneurship and government business enterprise : the pre-paradigmatic dance of the chameleon. Journal of Business Research, 52(1), 133-143. https://doi.org/10.1108/17515631011026380

Awang, A., Khalid, S. A., Yusof, A. A., Kassim, K. M., Ismail, M., Zain, R. S., \& Madar, A. R. S. (2009). Entrepreneurial Orientation and Performance Relations of Malaysian Bumiputera SMEs: The Impact of Some Perceived Environmental Factors. International Journal of Business and Management, 4(9). https://doi.org/10.5539/ijbm.v4n9p84

Bernama. (2014). EKUINAS UNDERTAKES RM400 MILLION INVESTMENT TO FURTHER EXPAND ITS OIL \& GAS AND F \& B PORTFOLIO.

Bernama. (2018). Ekuinas ready to acquire GLC subsidiaries. Bernama, pp. 5-6.

Buang, N. A., \& Yusof, Y. M. (2006). Motivating factors that influence class F contractors to become entrepreneurs. Jurnal Pendidikan Malaysia (Malaysian Journal of Education), 31(1), 107-121.

Chan, Paul, Horii, \& Kenzo. (1986). Impact of the new economic policy on the Malaysian economy: with special reference to ownership and control. Tokyo: Institute of Developing Economies.

Chin, J., \& Dosch, J. (2015). Malaysia Post Mahathir -.

Constitution, F. (2010). FEDERAL CONSTITUTION 1957. In The Commissioner of Law Revision, Malaysia. https://doi.org/JW516221 18-09-2010

Economic, P. U. (2001). The Malaysian Economy in 2001.

Economy, P. U. E. (2015). Malaysia: Changing Needs of Statistics Towards Becoming an Advanced Nation.

EKUINAS, R. A. (2014). DELIVERING PERFORMANCE. EKUITI NASIONAL BERHAD (EKUINAS).

Ghosh, B. C., Liang, T. W., Meng, T. T., \& Chan, B. (2001). The key success factors, distinctive capabilities, and strategic thrusts of top SMEs in Singapore. Journal of Business Research, 51(3), 209-221. https://doi.org/10.1016/S01482963(99)00047-8 
Hamidon, I. N. binti, Suhaimie, Yunoh, M. H., Naqib, M., \& Mat Hashim, H. binti. (2017). Entrepreneurial Intention Among Employees: an Insight of Entrepreneurial University in. 1-13.

Hamidon, S. (2008). The Development of Malay Entrepreneurship in Malaysia (Vol. 16). https://doi.org/10.1080/19761597.2008.9668649

Hashim, A., Che, O. C. M. Z., Hamzah, M. S. G., \& Umar, A. (2018). Leadership Behaviour, Entrepreneurial Orientation and Organisational Performance in Malaysian Small and Medium Enterprises. International Business Research, 11(9), 37. https://doi.org/10.5539/ibr.v11n9p37

Hung, D. K. M., \& Effendi, A. A. (2011). A PRELIMINARY STUDY OF TOP SMEs IN MALAYSIA: KEY SUCCESS FACTOR VS GOVERNMENT SUPPORT PROGRAM. JOURNAL OF GLOBAL BUSINESS AND ECONOMICS, 2(1), 48-58.

Lukas, S., \& Basuki, B. (2015). Implementation of Good Corporate Governance. The International Journal of Accounting and Business Society 47, 23(1), 1-25.

Ministry, F. M. (2017). Economic Management and Prospects. Economic Report 2017/18, 9-10. Retrieved from http://www.treasury.gov.my/index.php?option=com_content\&view=article\&id =6442: economic-report-2015-2016\&catid=262\&ltemid=2478\&lang=en

Mohamed, Z., Rezai, G., Shamsudin, M. N., \& Mahmud, M. M. az. (2012). Enhancing young graduates' intention towards entrepreneurship development in Malaysia. Education and Training, 54(7), 605-618. https://doi.org/10.1108/00400911211265648

Muhammad, K., Abu, H. M. I., Jamal, A. N. S., \& Adel, A. (2011). Challenges faced by the Small and Medium Enterprises (SMEs) in Malaysia: An Intellectual Capital Perspective. International Journal of Current Research, 3(2010), 398-401.

OPP3. (2001). THE THIRD OUTLINE PERSPECTIVE PLAN 2001-2010.

Ranjit, G. K. T. P. (1987). Tycoon on a Tightrope. Singapore: Sterling Corporate Services.

Rostamzadeh, R., Ismail, K., \& Bodaghi Khajeh Noubar, H. (2014). An application of a hybrid MCDM method for the evaluation of entrepreneurial intensity among the SMEs: A case study. Scientific World Journal, 2014. https://doi.org/10.1155/2014/703650

Sabiu, I. T., Abdullah, A. A., \& Amin, A. (2017). Impact of Motivation and Personality Characteristics on Bumiputeras' Entrepreneurial Persistence in Malaysia. Journal of Developmental Entrepreneurship, 22(02), 1750009. https://doi.org/10.1142/s1084946717500091

Schaper, M. (2010). Making Ecopreneurs : Developing Sustainable Entrepreneurship. British Library Cataloguing in Publication Data, (February), 1-321. https://doi.org/10.1108/13552551211228052

SME, C. (2019). SME Integrated Plan of Action (SMEIPA).

SME, R. A. (2016). SME Annual Report 2015/16. In SME Annual Report 2015/2016. Retrieved from http://www.smecorp.gov.my/images/Publication/Annualreport/SME AR 2015-16 English Final web.pdf

Soon, L. G., \& Huat, T. L. (1999). Determinants of compensation structure for salespeople in the retail clothing industry in Singapore: An agency theory perspective. Pacific Asia Journal of Management.

Thoburn, J., \& Snodgrass, D. R. (2006). Inequality and Economic Development in 
Malaysia. The Economic Journal, 91(364), 1071. ttps://doi.org/10.2307/2232532

Whah, C. Y., \& Guan, A. C. (2017). Malaysia's Protracted Affirmative Action Policy and the Evolution of the Bumiputera Commercial and Industrial Community. Journal of Social Issues in Southeast Asia, 32(2), 336-373. https://doi.org/10.1355/sj32$2 d$

Whah, C. Y., \& Guan, B. T. C. (2015). Malaysia' s Protracted Affirmative Action Policy and the Evolving Bumiputera Commercial and Industrial Community. ISEAS Economics Working Paper, 37.

Wiklund, J. (1998). Small Firm Growth and Performance Entrepreneurship and Beyond. Doctoral Thesis - Jönköping International Business School, 1-361.

Zulkifli, C. M., Omar, C., Mohd, N., \& Azmi, N. (2015a). Factors Affecting the Success of Bumiputera Entrepreneurs in Small and Medium Enterprises (SMEs) in Malaysia. International Journal of Management Science And Business Administration, 1(9), 40-45. https://doi.org/dx.doi.org/10.18775/ijmsba.18495664-5419.2014.19.1004

Zulkifli, C. M., Omar, C., Mohd, N., \& Azmi, N. (2015b). Factors Affecting the Success of Bumiputera Entrepreneurs in Small and Medium Enterprises (SMEs) in Malaysia. International Journal of Management Science And Business Administration, 1(9), 40-45. https://doi.org/dx.doi.org/10.18775/ijmsba.18495664-5419.2014.19.1004 